\title{
LA.UR. $90-4095$
}

CONF-4008173--1

LA-UR- $-90-4095$

DE91 004870

Los Alamos National Laboratery is operated by the University of California for the United States Department of Energy und r contract W-7405. ENG.36

\section{TTTLE: SMALL-ANGLE NEUTRON SCATTERING AT PULSED SPALLATION SOURCES}

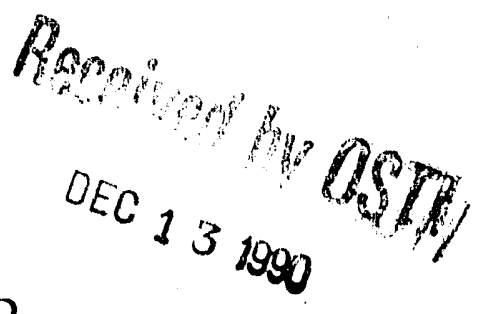

Aurhor(s): Philip A. Seeger and Rex P. Hjelm, Jr., LANSCE

submitred to: Journal of Applied Crystallography, 8th International

Conference on Small-Angle Scattering, Lenvan,

Belgium, August 6-10, 1990

By aceptance of this article, the publisher recognizes that the U.S. Government retains a nonexclusive, royalty-free license to publish or reproduce the publishad form of this contribution, or to allow othens to do so, for U.S. Government purpexics.
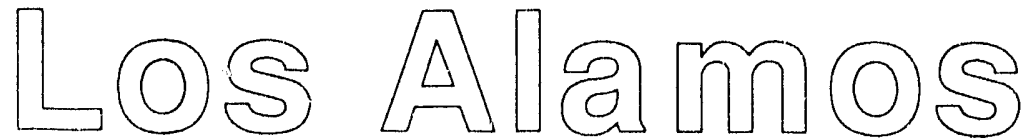

\section{Los Alamos National Laboratory Los Alamos, New Mexico 87545}

This report was prepared as an account of work sponsored by an agency of the United States

IORMNO 836 R4 ST NO 2629
Government. Neither the United States Government nor any agency thereof, nor any of their employees, makes any warranty, express or implied, or assumes any legal liability or responsibility for the accuracy, completeness, or usefulness of any information, apparatus, product, or process disclosed, or represents that its use would not infringe privately owned rights. Reference herein to any specific commercial product, process, or service by trade name, trademark, manufacturer, or otherwise does not necessarily constitute or imply its endorsement, recommendation, or favoring by the United States Government or any agency thereof. The views and opinions of authors expressed herein do not necessarily state or reflect those of the United States Government or any agency thereof.

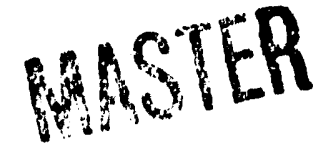




\title{
SMAILL-ANGLE NEUTRON SCATTERING AT PULSED SPALLATION SOURCES
}

\author{
P. A. SEEGiER and R. P. HJELM, JR. \\ Manuel Lujan Jr. Neutron Scattering C'enter \\ Los Alamos National Laboratory \\ Los Alamos, NM 87545 USA
}

\begin{abstract}
The importance of small-angle neutron scattering (SANS) in biological, chemical, physical, and engineering research mandates that all intense neutron sources be equipped with SANS instruments. Four existing instruments are described, and the general differences between pulsed-source and reactor-based instrument designs are discussed. The basic geometries are identical, but dynamic range is achieved by using a broad hand of wavelengths (with time-of-flight analysis) rather than by moving the detector. This allows a more optimized collimation system. Data acquisition requirements at a pulsed source are more severe, requiring large, fast histogramming memories. Data reduction is also more complex, as all wavelength-dependent and angle-dependent backgrounds and non-linearities must he accounted for before data can be transformed to intensity vs. Q. A comparison is shown hetween the Los Alamos pulsed instrument and D-11 (Institute Laue-Langevin), and examples from the four major topics of the conference are shown. The general conclusion is that reactor-based instruments remain superior at very low $Q$ or if only a narrow range of $Q$ is required, but that the current generation of pulsed-source instruments is competitive at moderate $\mathrm{Q}$ and may be faster when a wide range of $\mathrm{Q}$ is required. In principle, a user should choose which facility to use on the basis of optimizing the experiment; in practice the tradeoffs are not severe and the choice is usually made on the basis of availability.
\end{abstract}




\section{Introduction}

Small-angle scattering of neutrons and $X$-rays has proven to he an important methoul of probing structures on length scales of tens to hundreds of Ångstroms; the fact that this is the eighth conference of this series devoted to the techniques of small-angle scattering attents to that importance. The diversity of scientific fields addressed in these proceedingsincluding hiological structures, polymer conformation and thermodynamics, colloid structure. metallurgy, critical phenomena, etc.--further emphasizes the importance. The resulting demand for beam time (especially for neutrons, which are only available at large facilities) has led to the development of small-angle scattering instruments at a variety of sources, including pulsed spallation neutron sources. This report deals only with neutron small-angle scattering (SANS), and specifically with the four existing instruments at pulsed spallation sources.

The time-of-flight (TOF) technique, made easy by the pulsed nature of the source, is essential in order to achieve adequate count rates by using as much of the low-energy neutron spectrum as possible. There are however outstanding problems in data handling, because approximations used in data reduction at reactors do not necessarily work in TOF instruments. Considerable progress has been made in resolving these questions, so that data rates and quality at high-flux spallation sources are now comparable to those at high-flux reactor sources. The issues associated with TOF-SANS instruments are important: first, they require a careful look at the assumptions associated with the reduction of SANS data in general; second, the use of TOF may also be beneficial at reactor sources; and third, present political and economic realities make it likely that new neutron sources will be of the pulsed spaliation type. Thus the application of TOF to SANS will be of increasing importance.

There are special considerations required for using TOF, described in the following section. Section III reviews existing instruments with emphasis on how the design concepts of SANS using TOF differ from monochromatic sources. Data acquisition and reduction are more complex than at reactors, as discussed in detail in section IV; section V gives a detailed 
example and compares results with those from a reactor SANS. Finally a number of examples from the Los Alamos instrument are shown.

\section{Special Considerations for the Time-Of-Flight Method}

The design and use of SANS at spallation sources must take account of the characteristics of the TOF method, in which the time of flight $t$ from the source pulse to the detector is used to tag each recorded neutron with its wavelength $\lambda$ hy the relation

$$
\lambda=\frac{h}{M_{n}} \frac{t}{\left(L_{1}+L_{2}\right)}
$$

where $h / M_{n}=0.0003956 \AA-m-s^{-1}$ is Planck's constant over the neutron mass, and $L_{1}+{ }^{+}$, is the total length of the instrument. A limit on the length is imposed by the "frame overlap" condition; i.e., the wavelength for which the flight time is equal to the time between source pulses. The contributions to the variance of the momentum transfer $(Q)$ due to the spread of escape times from the moderator and due to the binning of time into discrete slices in the datit acquisition system are generally much smaller than the corresponding term for velocity selectors or monochromators.

The neutron spectrum at spallation sources is hotter than at reactors hecause the moderators used must be thinner to preserve good pulse characteristics. This makes the instruments most useful in the large- and mid-Q ranges. The greatest advantage of a pulsed source is its ability to use the broad spectrum to achieve a large dynamic range in $\mathrm{Q}$ with a single geometry of the instrument. The second major advantage also follows from the fived geometry, broad-band nature of the data recording: decisions concerning tradeoffs hetween intersity and precision in $\mathrm{Q}$ can be made after the experiment is completed, arid data reduction can be optimized to fit the requirements of the analysis.

Several disadvantages must be dealt with, however. 1) Limitations on instrument length make very low-Q measurements reliant on long wavelength neutrons, but the undermoderated sources have relatively low flux in this region. Further, multiple scattering 
associated with the large cross sections at long wavelengths can introduce additional problems. Instruments at high flux reactors may he more appropriate at very low $(Q$, although $Q$ as small as $0.0(0) 2 \AA^{-1}$ has heen demonstrated on TOF instruments for strong scatterers (Seeger, Hjelm, and Nutter 1990)). 2) The brightness of the source at high neutron energies, the presence of $\gamma$-radiation associated with spallation, and high instantaneous data rates can overwhelm standard gas detectors. Thus the ultimate potential of these instruments cannot he reached without further developments in detector technology (Convert and Forsyth 198.3). Filters, choppers, and bent bean guides can be used to reduce some of these problems. 3) Breakdown of the quasi-elastic assumption inherent in SANS data analysis potentially gives rise to extreme difficulties in a TOF instrument, particularly in materials with a high density of states in the energy range of the incident neutron beam (Hjelm 1988). 4) The data sets are taken in three dimensions, and thus can be extremely large, necessitating the use of high speed processors and computers for data transfer and reduction. Special sof tware packages must be provided (Hjelm and Seeger, 1989) to assist the user in reducing the data to a simple form suitable for analysis. The process of data reduction will be discussed in section IV.

\section{A Survey of Existing Pulsed-Source Instruments}

The instruments themselves are quite similar to reactor-based instruments, for which many articies concerning design concepts have been published. Issues associated with the design of pulsed-source instruments have been described by Crawford and Carpenter (1988). Other reports dealing with specific instruments include Borso, Carpenter, Williamson, Holmblad, Mueller, Faber, Epperson, and Danyluk (1982); Seeger, Williams, and Trewhella (1986); Ishikawa, Furusaka, Niimura, Arai, and Hasegawa (1986); Heenan (1987); Hjelm (1988); and Seeger et al. (1990).

Table I lists some characteristics of the Low-Q Diff ractometer (LOQ) at ISIS (Rutherford Appleton Laboratory, UK), the Low-Q Diffractometer (LQD) at LANSCE (I.0) Alamos National Laboratory, USA), the Small-Angle Diffractometer (SAD) at IPNS 


\section{PULSED-SOURCE SMALL-ANGLE NEUTRON INSTRUMENTS}

\begin{tabular}{|c|c|c|c|c|}
\hline & LOQ & LQD & $S A D$ & SAN \\
\hline Name & Low-Q Diffractometer & Low-Q Diffractometer & $\begin{array}{l}\text { Small-Angle } \\
\text { Diffractometer }\end{array}$ & $\begin{array}{l}\text { Small-Angle Neutron } \\
\text { Instrument }\end{array}$ \\
\hline Location & $\begin{array}{l}\text { Rutherford-Appleton } \\
\text { Laboratory, U.K. }\end{array}$ & $\begin{array}{l}\text { Los Alamos National } \\
\text { Laboratory, U.S.A. }\end{array}$ & $\begin{array}{l}\text { Argonne National } \\
\text { Laboratory, U.S.A. }\end{array}$ & $\begin{array}{l}\text { National Laboratory } \\
\text { for High-Energy } \\
\text { Physics, Japan }\end{array}$ \\
\hline Contact & R. Heenan & $\begin{array}{l}\text { P. Seeger } \\
\text { R. Hjelm }\end{array}$ & $\begin{array}{l}\text { E. Epperson } \\
\text { P. Thiyagarajan }\end{array}$ & M. Furusaka \\
\hline$\lambda$ & $2-10 \AA$ & $0.2-15 \AA$ & $0.5-14 \AA$ & $3-11 \AA$ \\
\hline$\Delta \lambda / \lambda$ & $2 \%$ & $1.6 \%$ & $<5 \%$ & $\sim 2 \%$ \\
\hline$L_{1}$ & $11.1 \mathrm{~m}$ & $8.40 \mathrm{~m}$ & $7.5 \mathrm{~m}$ & $19 \mathrm{~m}$ \\
\hline$L_{2}$ & $4.4 \mathrm{~m}$ & $4.32 \mathrm{~m}$ & $1.5 \mathrm{~m}$ & $1-5 \mathrm{~m}$ \\
\hline BeamSize & $\begin{array}{l}8 \mathrm{~mm} \varnothing \\
\text { (typical) }\end{array}$ & $\begin{array}{l}9 \mathrm{~mm} \varnothing \text { or } \\
22 \times 22 \mathrm{~mm}^{2}\end{array}$ & $12 \times 12 \mathrm{~mm}^{2}$ & \\
\hline Detector & $\mathrm{BF}_{3}$ Leti & ${ }^{3} \mathrm{He} \mathrm{Risö}$ & ${ }^{3}$ He Ordela & multiple linear ${ }^{3} \mathrm{He}$ \\
\hline Size & $64 \times 64 \mathrm{~cm}^{2}$ & $59 \mathrm{~cm} \varnothing$ & $19 \times 19 \mathrm{~cm}^{2}$ & $60 \times 62 \mathrm{~cm}^{2}$ \\
\hline$\Delta \theta$ & 0.002 & 0.001 & 0.002 & 0.002 \\
\hline Q Range & $0.006-0.22 \AA^{-1}$ & $0.003-0.5 \AA^{-1}$ & $0.005-0.35 \AA^{-1}$ & $0.003-4 \AA^{-1}$ \\
\hline Flux & $\sim 10^{5} \mathrm{n} / \mathrm{cm}^{2} / \mathrm{s}$ & $\sim 10^{5} \mathrm{n} / \mathrm{cm}^{2} / \mathrm{s}$ & $\sim 10^{5} \mathrm{n} / \mathrm{cm}^{2} / \mathrm{s}$ & $\sim 10^{5} \mathrm{n} / \mathrm{cm}^{2} / \mathrm{s}$ \\
\hline
\end{tabular}


(Argonne National Laboratory. USA), and the Small-Angle Neutron Scattering instrument (SAN) at KENS (National Laboratory for High-Energy Physics, Japan). The numbers in the table may represent typical, average, or approximate values, and are not intended for intercomparison of the instruments. They may be compared in general with reactor-based instruments, as tabulated in a recent report from Oak Ridge National Laboratory (McElhaney and Vandermolen 1990).

The first issue in instrument design is the target-reflector system and the moderator. Cryogenic hydrogenous moderators are used to shift the neutron energy spectrum to a more useful wavelength range. Moderators for TOF must be thin so that pulse width of emitted neutrons is narrow enough to provide adequate time resolution. The resulting undermoderated spectrum has a large epithermal component. However, time resolution is not so important for SANS, as the overall resolution is dominated by geometric factors. Specialized moderators for low $-\mathrm{Q}$ measurements would sacrifice time resolution to ohtain higher intensities at long wavelengths. Work on moderator design is in progress at KENS and at LANSCE (Kiyanagi and Watanabe 1990; Russell 1990), and future instruments will undoubtedly see improved performance. The need to have a specialized moderator for SA.VS at a pulsed source contrasts with reactors, where the same moderator serves very different types of instruments.

Another source-related issue is the initial incident flux of hot neutrons and $y$-radiation. This hot component will interact in the collimation, shielding, and with the sample itself, eventually moderating and producing a background at later times in the detector. Target and moderator design, such as the flux-trap arrangement at the LANSCE source (Russell, Gilmore, Robinson, Legate, Bridge, Sanchez, Brewton, Woods, and Hughes 1989), can mitigate this problem, but other strategies are required to reduce the hot component to a level which doesn't interfere with measurements. For example, LOQ uses a beam bender with a cutoff wavelength of $2 \AA$ to remove the instrument from the line of sight of the source (Heenan 1987). On LQD and SAD, cold single-crystal MgO filters are used to remove 
neutrons below about $1 \AA$ (Epperson, Worcester, Thiyagarajan, Klippert, Crawford, and Carpenter 1987). A chopper may also be used to absorb the initial pulse. Removal of the hot component improves detector response, reduces effects of inelastic events in the sample (Hjelm 1988), and improves collimation by reducing the probability of penetration or scattering in the collimator materials. These are not issues at reactor sources because monochromatization removes the high-energy component.

Collimation may be by pinholes (LOQ and LQD) or by multiple converging soller slit assemblies (SAD and SAN). Another possibility is a multiple pinhole system, with intermediate beam scrapers to prevent cross talk (Glinka, Rowe, and LaRock 1986; Seeger 1986). Maximizing intensity for a given angular variance at the detector (or minimizing the variance for a fixed intensity) leads with complete generality to the criterion that the sizes of apertures must be proportional to the distance from the detector; i.e., all dimensions of the collimator converge to a point at the detector (Seeger 1980; Mildner and Carpenter 1984). Fixed collimation thus suggests a fixed detector location; dynamic range is achieved by using a wide wavelength band. This allows the full range to be measured in a single instrument configuration. The SAN has a moveable detector, which is optimized at $5 \mathrm{~m}$, and also several individual detectors at wide angles to extend its dynamic range even more. The detector on SAD may be moved off-axis to extend its dynamic range and improve precision at a given $Q$ (Hjelm 1987, 1988).

From Liouville's theorem (conservation of phase-space density), variance and intensity remain constant as the instrument length is changed if all other dimensions scale proportionally (Seeger 1986). Since the detector resolution contributes a term to the angular variance which does not scale, longer flight paths are preferred, up to the point at which the full extent of the source is being viewed or (in the case of pulsed instruments oniy) to the point where frame overlap truncates the desired wavelength range. Since the individual apertures of soller collimators are quite small, the collimator can be much shorter than a pinhole system, alleviating the frame overlap problem. However, extreme care must be used 
in choosing and fabricating the absorhing plates, since reflection prohability increal:en ats $:^{2}$ (Crawford, Epperson, and Thiyagarajan 1989). The LOQ and SAN instruments include mechanical choppers to absorb alternate beam pulses and double the frame width (and range of $\lambda$ ). Soller or multiple-aperture collimators increase total neutron current by increasing the available sample area, up to the limit at which the entire surface of the moderator is heing viewed.

Another feature of long flight times is the effect of gravity. In $50 \mathrm{~ms}$, a neutron falls $12 \mathrm{~mm}$, so long-wavelength (slow) neutrons follow arched trajectories through the instrument. For a monochromatic source the detector can be positioned where the heam is: for a white source the beam center changes with time of flight. On LQD, the exit aperture of the collimator is accelerated upward during each beam pulse so that the trajectories always converge to the center of the detector; this is called "dynamic gravity focusing" (Seeger et al. 1990).

Detectors for TOF instruments are identical to those used at reactors. There is a problem in that standard position-sensitive ${ }^{3} \mathrm{He}\left(\mathrm{or} \mathrm{BF}_{3}\right.$ ) detectors with traditional encoding schemes cannot handle the high instantaneous data rates of some experiments. Collimation must he stopped down for some strong scatterers, and (at least in the case of LQD) a live-time correction is required for all transmitted-beam measurements. Other detector problems include recovery from the initial high-energy pulse, and generally low efficiency at the sho-test wavelengths recorded. These problems must be solved for the full potential of spallation sources to be realized. Anger cameras using ${ }^{6} \mathrm{Li}$-doped scintillator glass are a possibility, but novel position-encoding schemes (e.g., Seeger 1984) must be developed to he able to make use of the intrinsically faster counting capability. Also, scintillators have an unfortunately high sensitivity to $\gamma$-radiation.

The final entries in Table I, flux, can not be compared to reactors (or even to each other) because the "quality" (or information content per neutron) is different on every 
instrument. The point of including the order-of-magnitude estimate is to bow that the timeaveraged neutron current at pulsed sources is quite low, so that no neutrons may he wasted.

\section{Data Acquisition and Reduction}

Figure 1 illustrates schematically hoth the mechanical simplicity and the electronic complexity of the LQD (henceforth taken to be "typical" of pulsed-source instruments). Vote specifically that the neutron position within the detector is digitized (in hardware) and pained directly to a histogramming memory (ina Fastbus crate) without use of any computer. This allows an instantaneous rate limited only by the detector encoding. The detector in digitized to $128 \times 128$ cells in as many as 256 time slices, so the maximum memory size for LQD is $128 \times 128 \times 2563$-byte words or 12 Mbyte. A computer has access to this memory in real time to provide displays as the data accumulate and also to compress and transfer data to the facilitywide archive. All data are preserved for every data run so that tradeoffs between resolution and intensity can be made during data reduction. The computer is part of an extensive network giving access and color graphics at remote locations.

Even if the histogram could be displayed as a function of three variables, it would not usually be meaningful; it must be reduced to a function of two (e.g., X-Y or R-t) or one variables, and eventually to scattering as a function of $Q$ in 2 - or 1-dimensions. The most direct rendition available in real time is a map of the detector summed over a few time slices; fig. 2 is such a plot for a sample of $\mathrm{C}_{12} \mathrm{E}_{6}$ (n-dodecylhexaoxyethylene glycol monoether) iri at lamellar phase. High-contrast colors are used to represent count rates per detector cell with at square-root intensity scale. This plot can be provided in real time and updated on demand. Radial averages may also be taken, and an approximate $Q$-scale can be generated corresponding to the mean wavelength of the chosen time slices. One-dimensional plots of the sum of the detector as a function of time slice and of the upstream and transmission monitor detectors are also available during acquisition. An early version of the general software package is lescribed in Hjelm and Seeger (199()). 


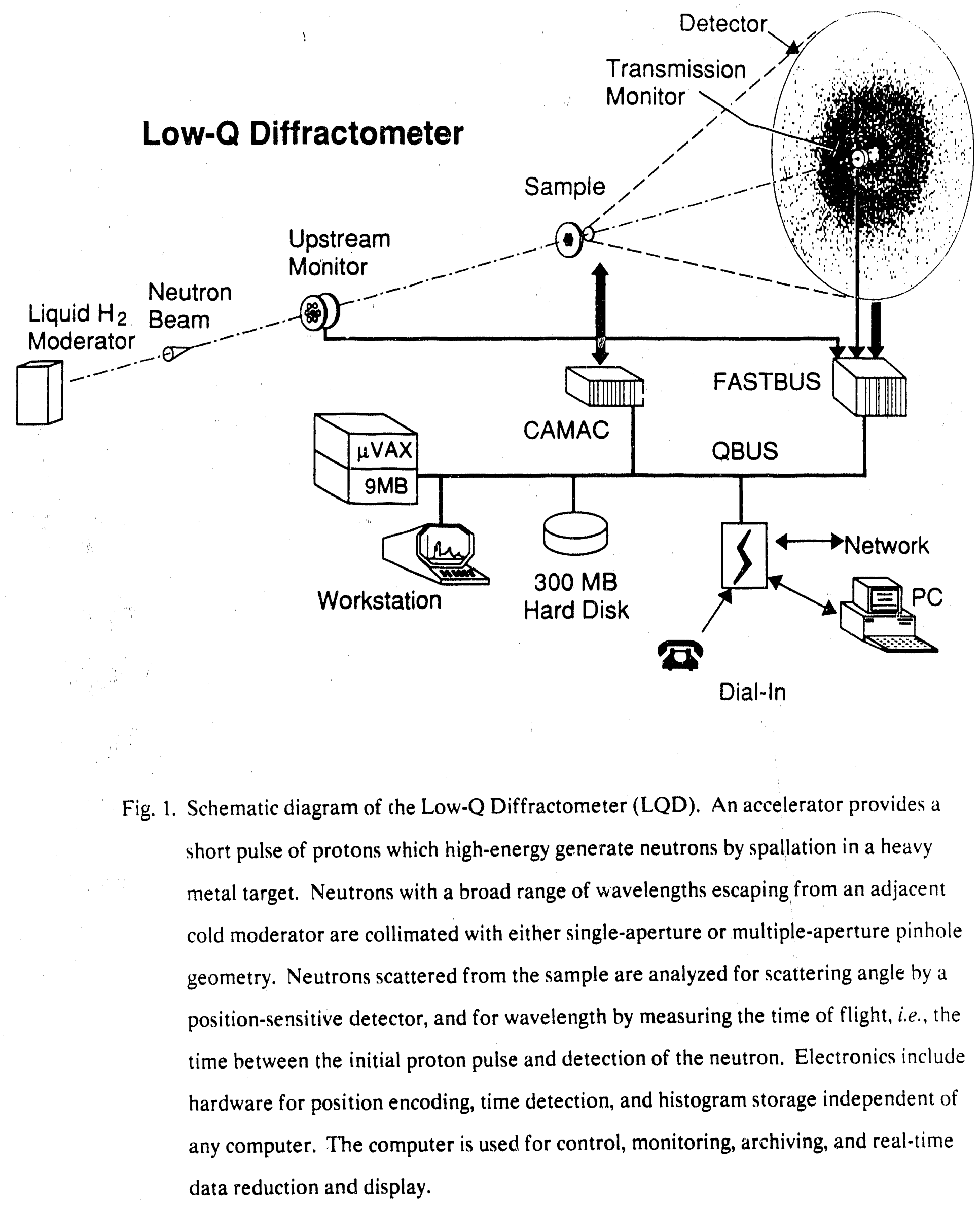


-

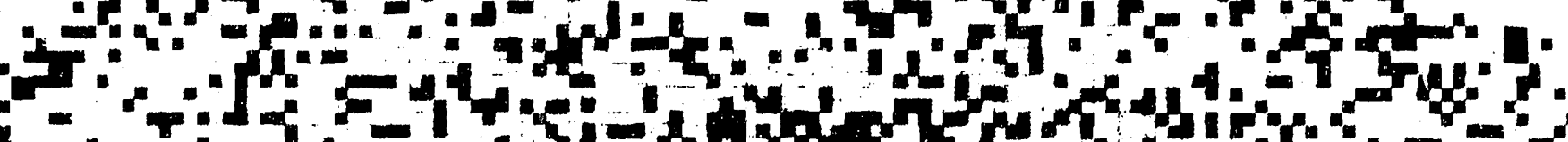

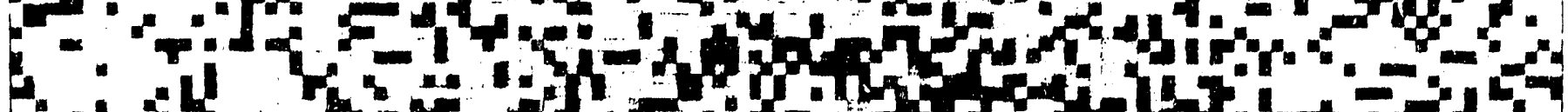

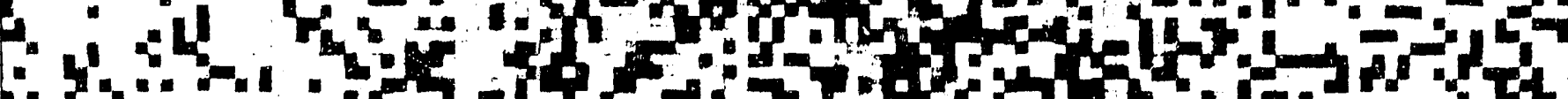
4.".

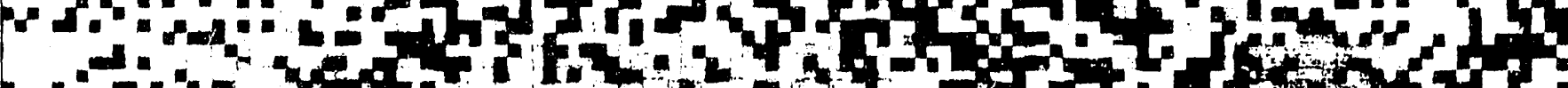
m.1.

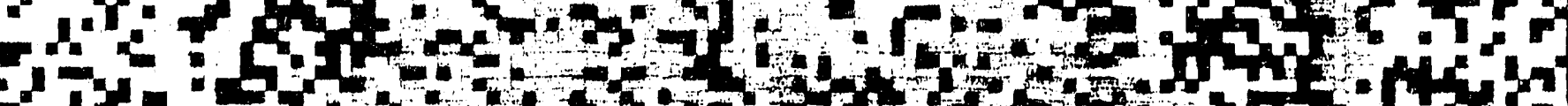

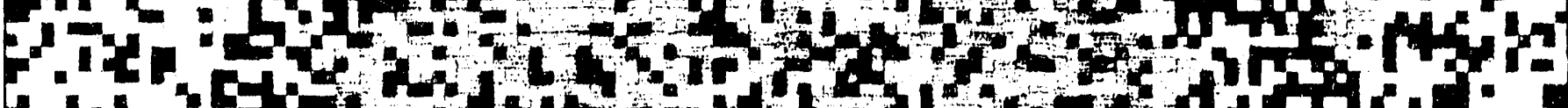

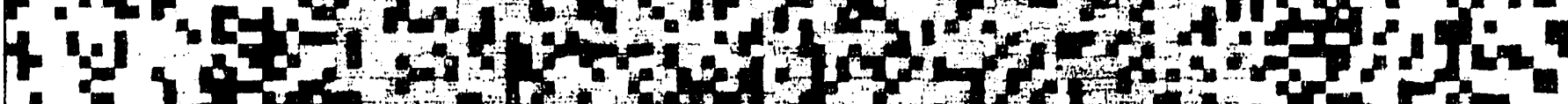

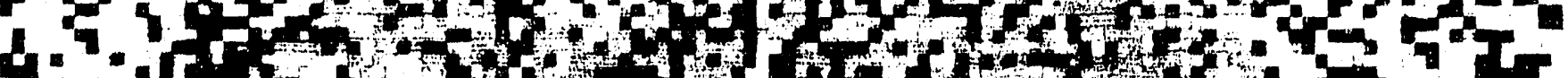

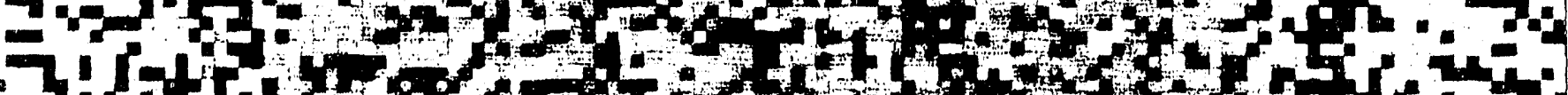

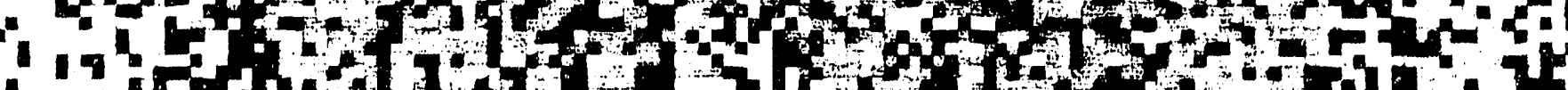

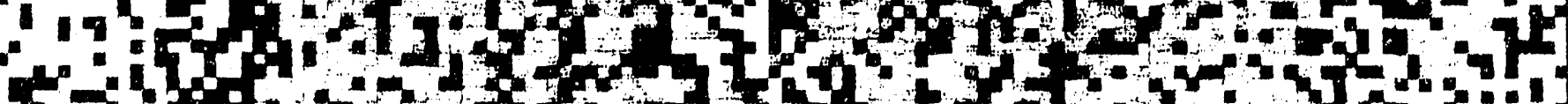

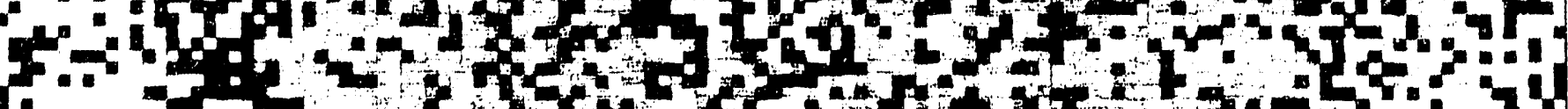

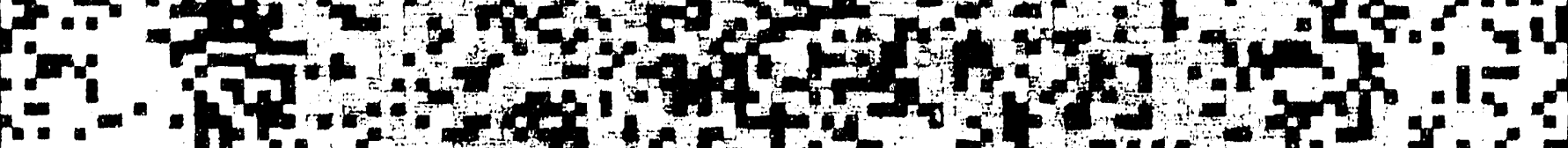

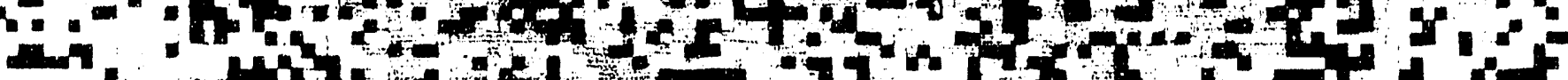

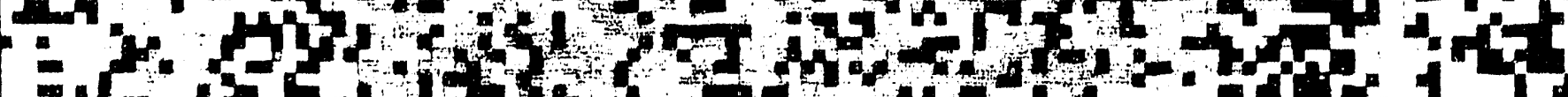

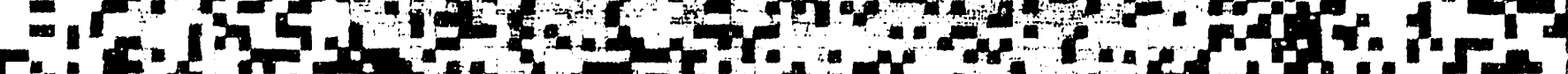

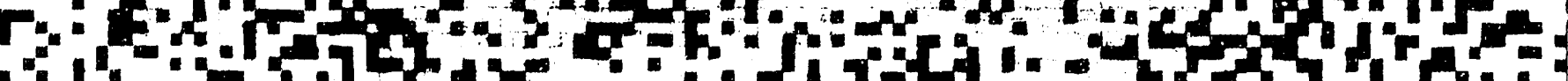

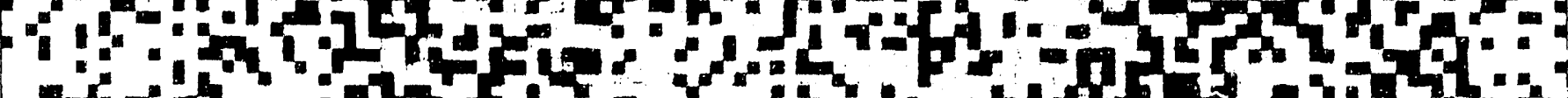

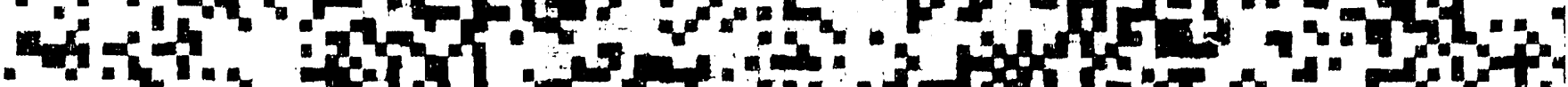

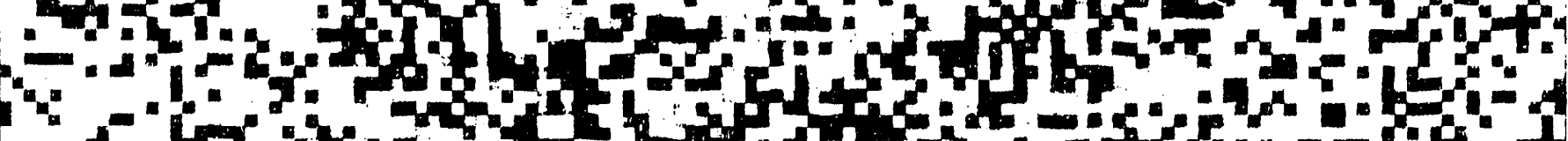

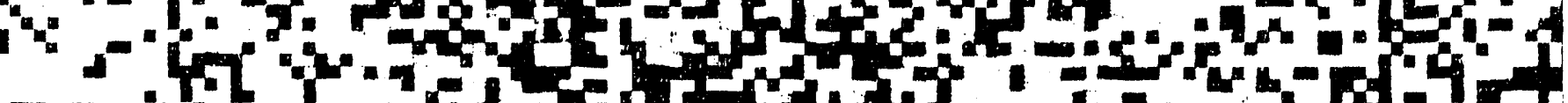

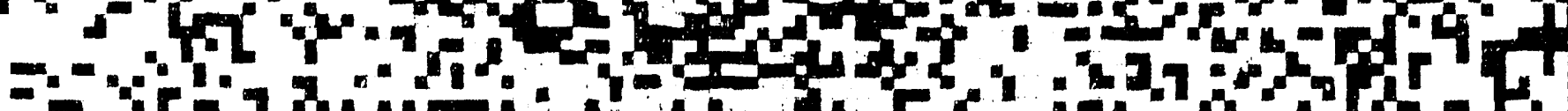

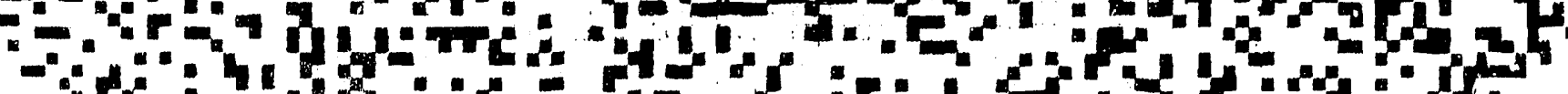
-1.

Fig. 2. Real-time display of intensity vs. detector position, for a sample of $\mathrm{C}_{12} \mathrm{E}_{6}$ (ndodecylhexaoxyethylene glycol monoether) in a lamellar phase. Six time slices are sumined, resulting in a $10 \%$ wavelength spread at a wavelength of $1.66 \AA$. Data collection time was 1 hour, and the maximum count per pixel is . 36. To obtain full statistics, all time slices are summed after conversion to $Q$ bins. Preferred alignment is evident in this sample. 
Data reduction for TOF requires rebinning of the $X-Y$ position histogram for earh time slice (wavelength) into vector (or radially averaged) Q-space in a manner which allows subsequent combining of the ime slices (Seeger and Pynn 1986, Hjelm 1986). The "huckets" in fig. 3 represent the cells of the ráw-data histogram (shown radially averaged for simplicity). For LQD, the radial scale is linear and the time scale is logarithmic. The rehinning problem is to transfer the neutrons from the buckets to Q-bins. If the neutrons are poured out, one bucket may contribute to several bins, leading to unwieldy correlations in the statistical error:, We therefore choose to drop the buckets into the bins telow their centers; this maintains Poisson statistics of the histogram, hut results in the variance of $Q$ in the resulting bins being greater than would be computed from the bin wi.dth alone. To mitigate the efiect of the rebinning on the resolution, we encode the detector into $X-Y$ bins which are much smaller than the geometric resolution of the instrument. Problems still arise when the buckets are considerably wider than the bins they drop into; inclusion of such data may compromise $Q$ resolution. As discussed fully in Hjelm (1988), the minimum time slice to be included is a function of the width of the Q-bin; a fixed bin width (linear Q-scale) thus corresponds in first order to a particular minimum t. When a logarithmic Q-scale is used, this is equivalent (because of the reciprocal relationship between time and radius in determining $Q$ ) to a fixed minimum radius on thie detector. We therefore use a logarithmic scale $(\Delta Q / Q=0.167)$ for the $\mathrm{Q}$-regime below $\mathrm{Q}=0.006 \AA^{-1}$ which is limited by a minimum inner radius, and a linear scale $\left(\Delta Q=0.001 \AA^{-1}\right)$ in the range up to $Q=0.04 \AA^{-1}$ where long wavelength-neutrons contribute maximum information. To improve statistics at higher $Q$, the scale then becomes logarithmic again $(\Delta \mathrm{Q} / \mathrm{Q}=\mathbf{0 . 0 2 5})$. Thus, the resolution criterion is that data from the inner portion of the detector should not be used in the high-Q regime and early time slices (short wavelengths) should not be included at low $Q$ (Seeger and Pynn 1986). The parameters of the Q-scale, and the criterion of bucket widths to be retained, may be adjusted during data reduction. In fig. 3, the buckets in the lower-left corner can be seen to be wider than the Qhins below them and would be deleted. 

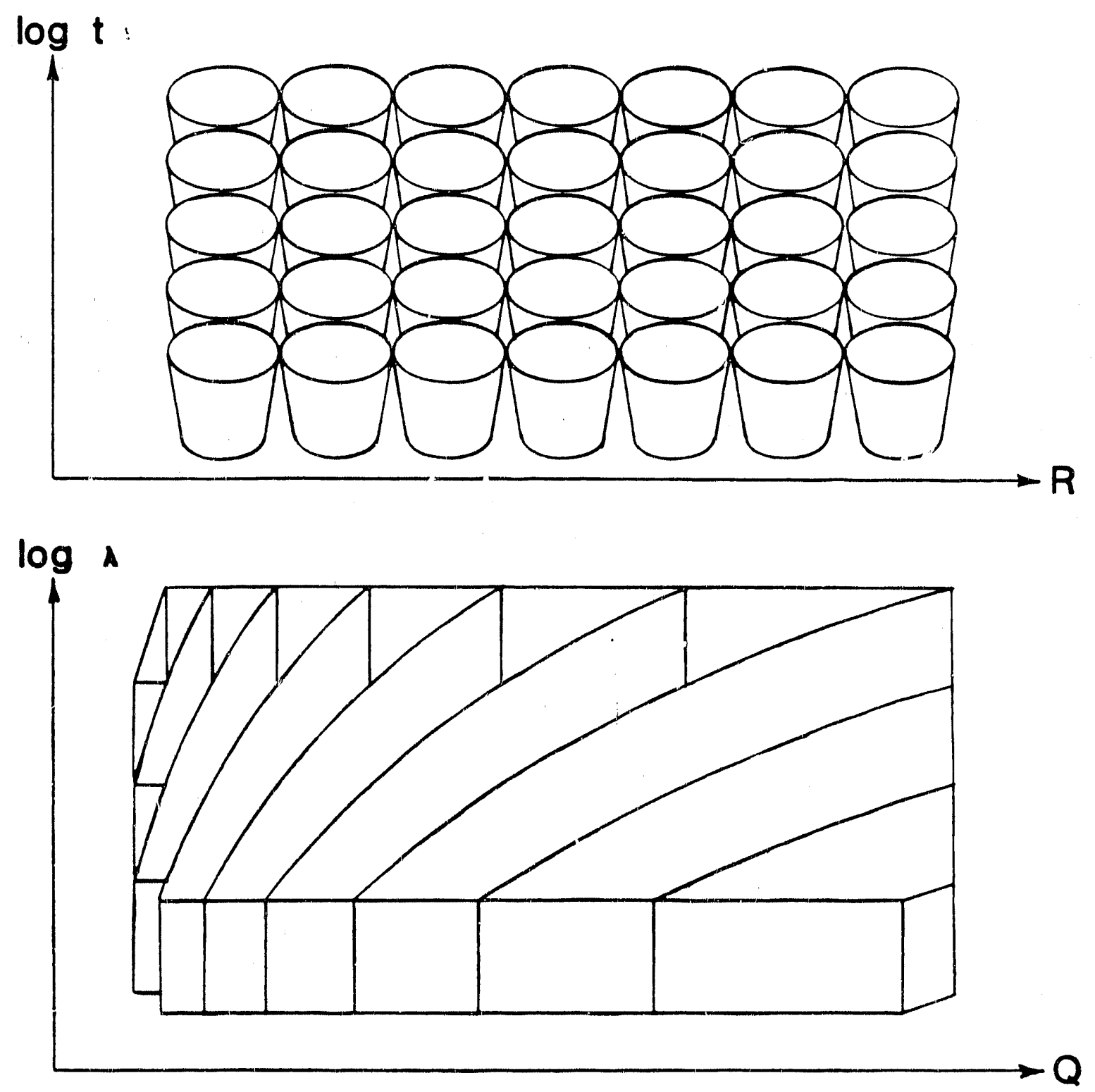

Fig. 3. Mapping of "buckets" in R-log(t) space into Q-bins. Neutrons from many time slices (wavelengths) detected at appropriate detector positions (scattering angles) contribute information to any value of $Q$. The buckets are defined during data acquisition, hut intensity/resolution tradeoffs are made after the experiment by definition of the $\mathrm{Q}$ bins and setting criteria for how much data to include from shorter wavelengths and/or smaller radii. See text for further details. 
Any corrections which are functions of time or of detector position must be mate hefore the data are rehinned. To first order, this means dividing each histogram element hy the transmitted neutron spectrum factor $\Phi\left(r_{i}\right)$ and hy the detector solid angle $\Omega\left(R_{j}\right)$, where indices i and i refer respectively to the specific time slice and pixel for annular zone, if radially averaged). The transmitted spectrum, $\Phi\left(t_{i}\right)$, is the number of countathle neutrom per time slice, and is either measured in the main detector and normalized to the same cotal incident flux as the scattering measurement, or else it is measured simultaneously with the scattering in the downstream transmission monitor and then corrected for the ratio of efficiencies of the detector and the monitor (Seeger et al. 199()). Second order correctiom tu the solid angle include detector nonlinearity and nonuniform efficiency, and small trigonometric corrections. If there is a significant blocked-beam signal, which is frequently true at spallation sources, it should in principle also be subtracted at this step; however in many cases the subsequent subtraction of a blank will correct adequately for the hlockedheam component. The absolute scattering probability in bucket (i,j) per unit solid angle in

$$
\frac{d P_{i j}}{d s z}=\frac{v_{i j}}{\Phi\left(t_{i}\right) \Omega\left(R_{j}\right)}
$$

where $v_{i j}$ is the number of neutrons in the hucket. Suppose this $(i, j)$-hucket fall, into hin $k$, which may represent a specific vector $Q$ or radially averaged $Q$. In principle all buckets contributing to this bin give the same $d P / d \Omega$ and hence can be averaged. In order (1) retain the information content of each hucket, we weight them according to the relative number of neutrons which should have heen detected, $\Phi\left(t_{i}\right) \Omega\left(R_{j}\right)$. Then

$$
\frac{d P\left(Q_{k}\right)}{d \Omega}=\frac{\sum^{N} N_{i j}}{\sum \Phi\left(t_{i}\right)} \Omega_{\Omega\left(R_{j}\right)}
$$

where the summations are over all $(i, j)$ which generate $Q_{k}$, and all relevant correctiom are made hefore summing. This form of the average accounts properly for empty buckets, and 
propagation of statistical errors is straightforward. To convert this absolute scattering probability to the more customary macroscopic differential cross section per unit volume $\mathrm{d} \Sigma / \mathrm{d} \Omega$ in units of $\mathrm{cm}^{-1}$, divide by the sample thickness in $\mathrm{cm}$.

In most instances the scattering of interest is obtained by subtraction of $\mathrm{dP} / \mathrm{d} \Omega$ for a blank (e.g., an empty cell, or a buffer solution). Great care is required at this step because hackground (blocked-beam) contributions have not been explicitly removed from the counts $\mathrm{N}_{i j}$ in eq. (3). If the scattering signal is large, or if the transmissions of the signal and the blank are alike, then the blocked-beam contribution will cancel. If however the scattered signal is very small or the transmissions are significantly different, then a correction term is required. The background can be measured by using a fairly thin (0.8- $\mathrm{mm}) \mathrm{Cd}$ absorher which allows the high-energy component of the beam to pass but completely absorbs those neutrons which have wavelengths belonging to the recorded TOF slices. Thus any observed counts represent neutrons which have been moderated in the shielding or other parts of the instrument after passing the sample position and are detected at later times. This is a major source of sample-independent trackground at LQD. To simplify propagation of statistical errors, the Cd-blocked beam signal is treated as a correction term, found by normalizing the $\mathrm{Cd}$ measurement to a pseudotransmission which is the harmonic difference of the two measured transmitted fluxes: $1 / \Phi_{C d}(t)=1 / \Phi(t)-1 / \Phi_{b}(t)$. Using the notation $(S) /(T)$ to represent the ratio of normalized sums in eq. (3), (B)/(BT) for the corresponding blank, and $(\mathrm{Cd}$ ) for the Cd data, all normalized to the same amount of incident beam, the corrected scattering probability is

$$
\frac{\mathrm{dP}}{\mathrm{d} \Omega}=\frac{(\mathrm{S})}{(\mathrm{T})}-\frac{(\mathrm{B})}{(\mathrm{BT})}-\left[\frac{1}{(\mathrm{~T})}-\frac{1}{(\mathrm{BT})}\right](\mathrm{Cd})
$$

\section{Sample Data Reduction}

As an example of normalization and rebinning, we use radially averaged data from Vycof glass sample PT-5, prepared by P. Thiyagarajan of IPNS for use at SAD and as a 
secondary vandard for intercalibration of various facilities. Ordinarily a uner would not halte to see any of the intermediate steps, but these plots are often useful for diagnantics.

This sample has a pore-pore correlation distance of ahout $310 \AA$, leading to a broal peath at $Q=0.12 \AA^{-1}$. In the wavelength range recorded, the peak forms an arc in the $R-\log (t)$ plot of fig. ta extending from the edge of the heamstop at early time slices to the outer edge of the detector at late times. This allows calibration of the non-linearity of the position encoding in the detector. Figure 5 shows the measured $\Phi(t)$; the data have heen corrected for rate-dependent dead time in the detector ( $15 \%$ at the peak), and the last 25 time slices have heen fitted by an exponential tail hecause the statistics on any one slice are low. The clata are normalized by the attenuation factor of the transmission mask used and hy the ratio of integrated proton currents, to give the total detectable number of neutrons which passed through the sample; note that detector efficiency cancels between the numerators and denominators of eqs. (2)-(3). Figure 6 shows the solid angle in each of the radial zones; open circles are uncorrected and filled circles are corrected for detector non-linearity, which is severe at the outer edge. Applying eq. (2) to fig. ta produces fig. th, still in R-log(t) spare. and conversion to $Q$ gives fig. 4c. It is clear that resolution broadens the peak and reduces its height in early time slices. Also, in the lower-left region where the radial hucket widths are considerably broader than the Q-bins, there are many bins which receive no huckets at all. The black line on fig. $+c$ indicates the approximate houndary at which bucket width eyuals bin width; only data ahove and right of this line are retained in computing the average $\mathrm{dP} / \mathrm{d} \Omega$ from ey. (3). All contours (color houndaries) should he vertical lines on this plot; the apparent residual errors of normalization or linearity also affect resolution in $Q$.

This sample is such a strong scatterer that the Cd-blocked heam correction is negligible. Averaging hy eq. (3), subtracting a similarly treated empty-cell data set, and dividing by the sample thickness of $0.159 \mathrm{~cm}$ gives the open circles in fig. 7 . which also includes data from the same sample taken at the $10-\mathrm{m}(*)$ and $5-\mathrm{m}(*)$ detector positiom an the D-1 1 instrument at the Institute Laue-Langevin (ILL). From this figure we judge the 
a)

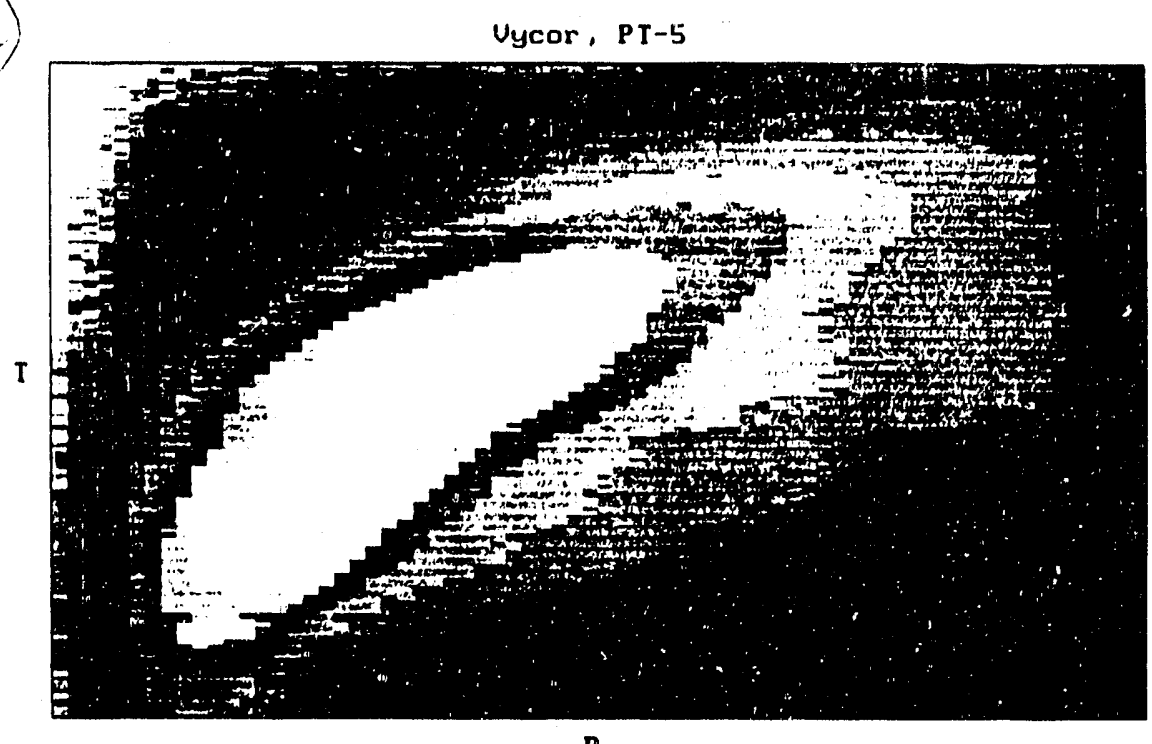

Uycor, PI-5

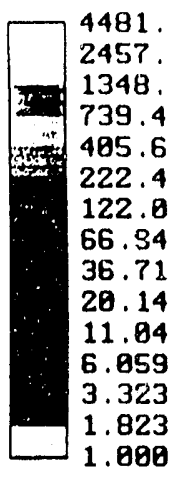

b)
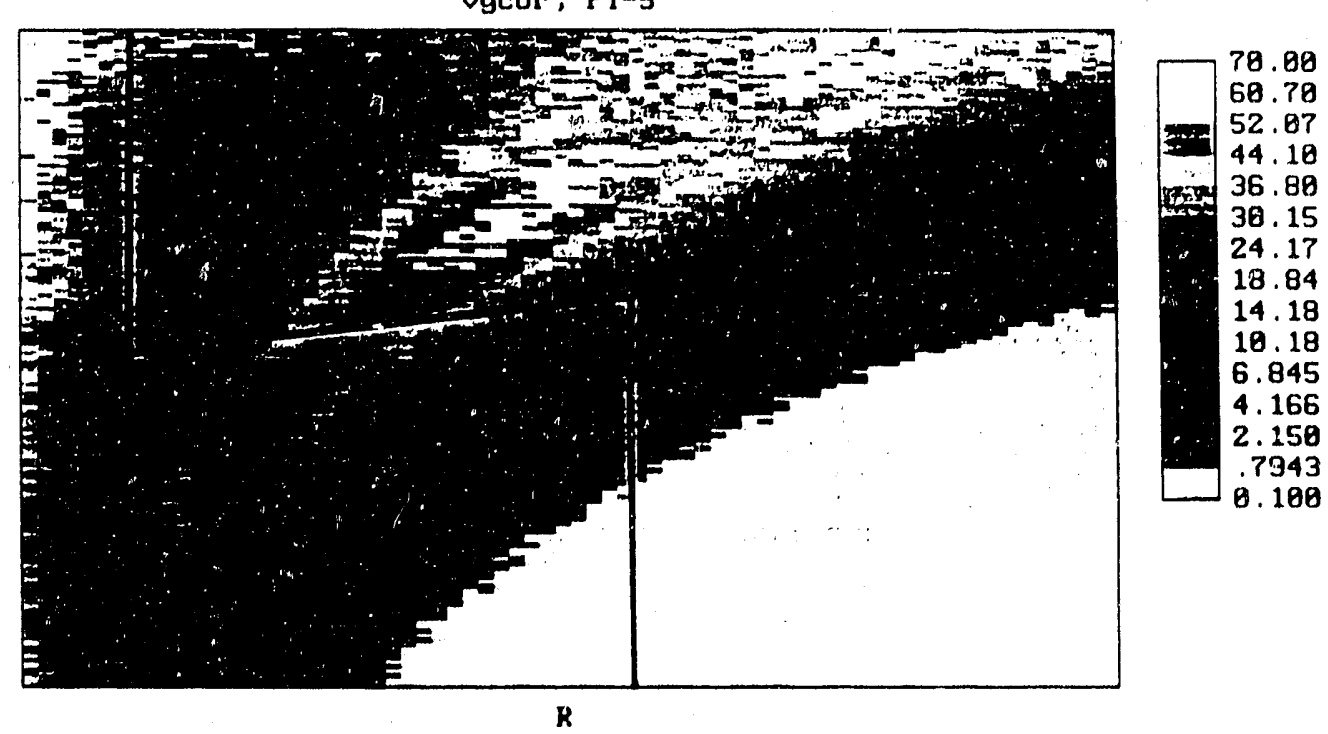

C)

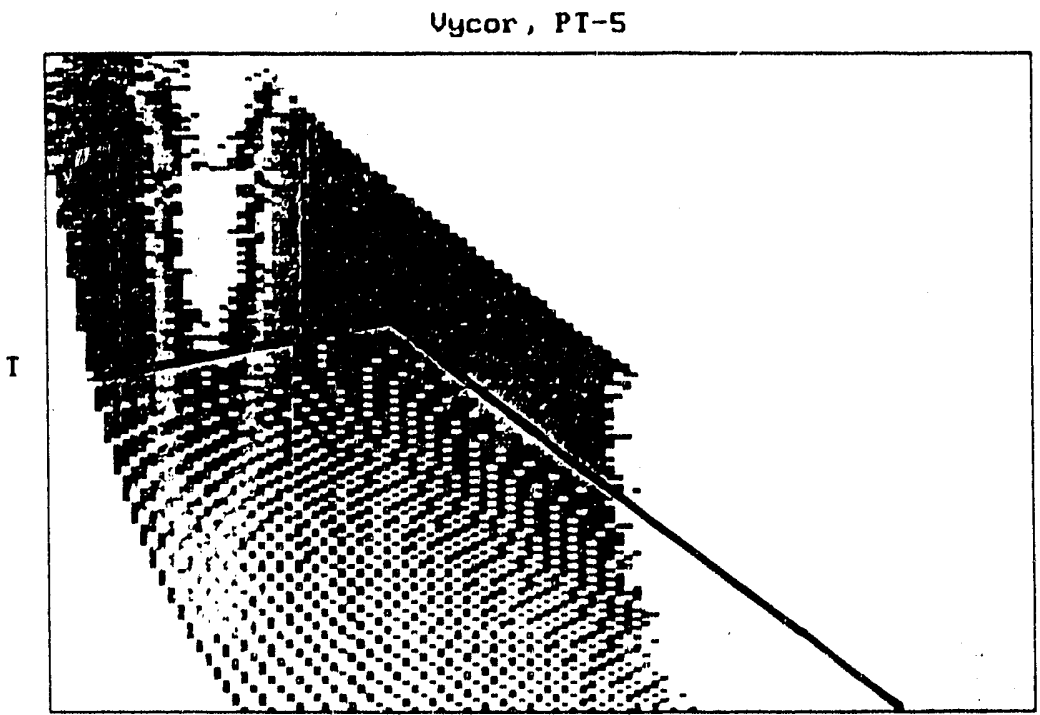

a

Fig. 4. Radially averaged data from Vycor sample PT-5, a) Raw data v's. detector radiun and time slice. Colors represent logarithm of intensity. h) Normalized hy number of neutrons per time slice and hy solid-angle per radial zone. Colors on a square-root 


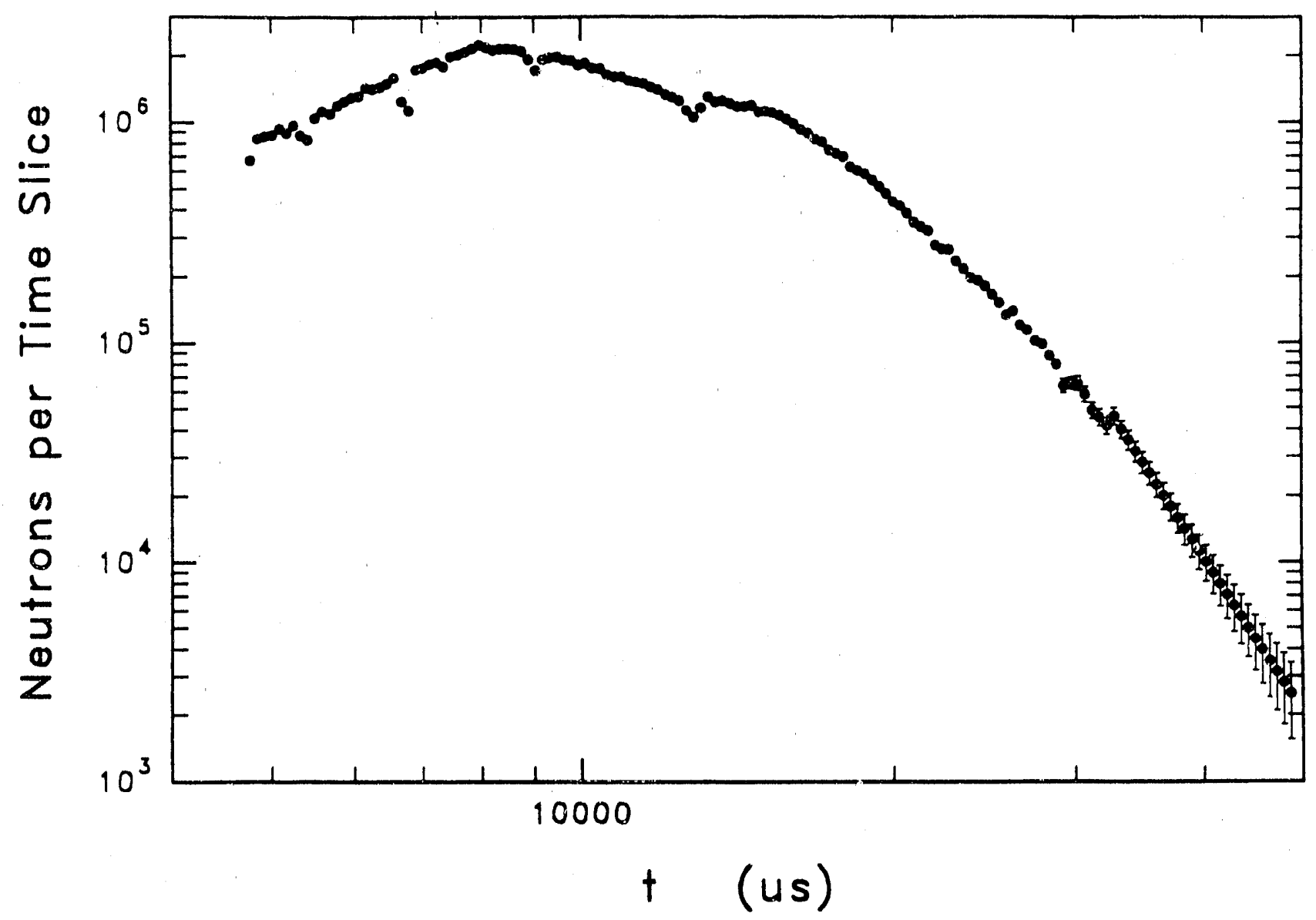

Fig. 5. Transmitted beam for sample PT-5, measured in the main detector by inserting an attenuating mask and removing the heamstop. A livetime correction proportional to instantaneous count rate (maximum ce rection 15\%) has been applied, and late time slices have been smoothed. Dips at 9000$) \mu \mathrm{s}$ and $13000 \mu \mathrm{s}$ are due to aluminum Bragg edges from the moderator can and detector window; earlier-time dips are from the single-crystal MgO filter used to remove shorter-wavelength neutrons from the heam 


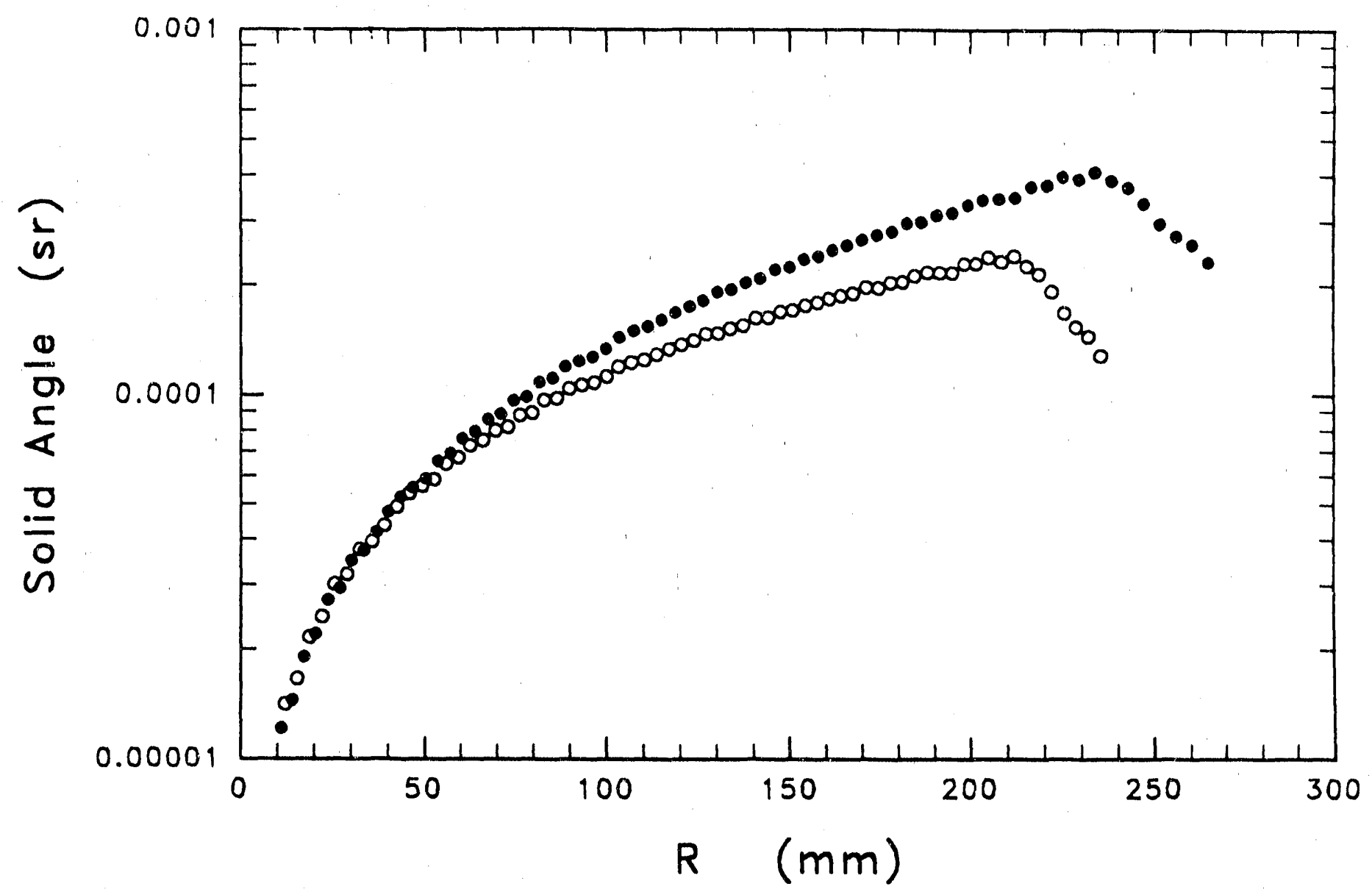

Fig. 6. Solid angle of radial zones in the detector, with linear calibration (o) or with quadratic correction $(\bullet)$ as determined by the requirement that the location of a peaked $\mathrm{I}(\mathrm{Q})$ function must be proportional to neutron wavelength. 


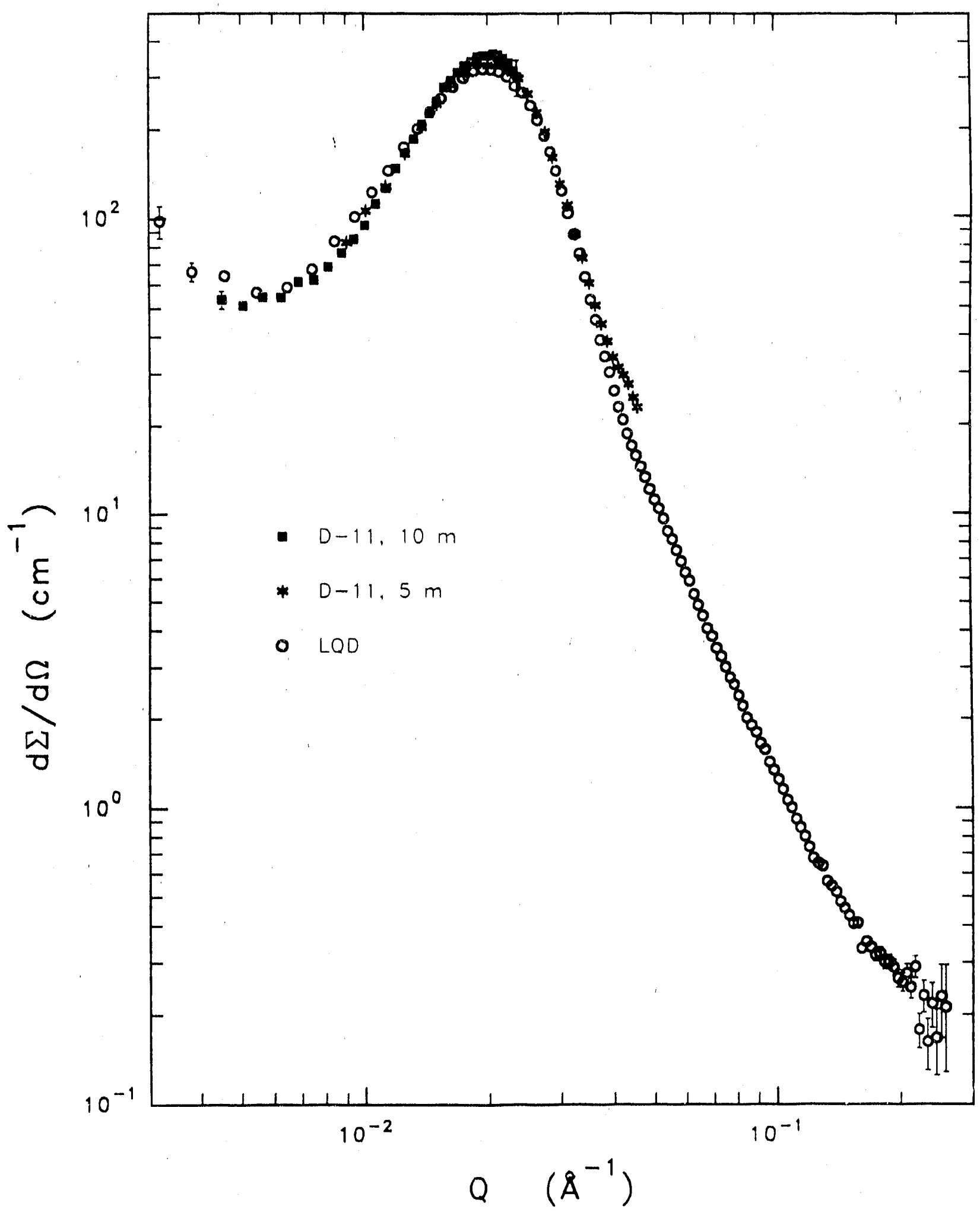

$\Gamma$

Fig. 7. Macroscopic scattering cross section for Vycor sample PT-5, as measured on LQD ( $\circ)$. Also shown are data from the same sample taken at the $10-\mathrm{m}(\cdot)$ and $5-\mathrm{m}(*)$ detector positions on the D-11 instrument at the Institute Laue-Langevin. From the quality of the data we judge the resolution to be equivalent to the 5-m position at $D-11$. Pulsedsource instruments are advantageous when a large dynamic range of $Q$ is required, since the entire range is collected with a single instrument setting. 
resolution to be equtivalent to the $5-m$ position at $D-11$, when data are reduced with the parameters ahove. By moving the line in fig. tc, or by making the Q-bins larger or umaller. one can trade intensity for resolution. The data acyuisition time for this experiment and its transmission was 3() minutes at a proton heam current of 8()$, 4 \mathrm{~A}$. To achieve the same statistics $(0.4 .5 \%)$ in an equal width $Q$-hin (0.0(0) $\left.\AA^{-1}\right)$ at a single value of $Q\left(0 .\left(1.30 \AA^{-1}\right.\right.$, al D-11 would take 9 minutes at the $5-m$ position. Thus it takes more than 3 times as long to get a data set at LQD, hut the $\log (Q j-r a n g e$ ohtained is nearly t times as wide. In this example. the exiended range allows determination of the power-law slope ahove $Q=0.0+5.5 \AA^{-1}$. When a constant incoherent contribution of $0.14 \mathrm{~cm}^{-1}$ is subtracted, the slope of $-.3 .360 \pm 0 .(006$ extends to the highest values of $Q$ measured.

Our general conclusion is that while present reactor sources are superior for measurements at very low $Q$ or over a narrow range of $Q$, the current generation of sma!langle instruments on pulsed spallation sources is competitive at moderate $Q$ and may he faster when a wide range of $\mathrm{Q}$ is required.

\section{Examples from Various Disciplines}

In keeping with the scientific diversity of science represented in these proceedings. examples have heen selected to represent the general fields of hiology, synthetic polymers. colloids, and materials science.

\section{A. Distance between antigen binding sites of immunoglobulin IgG1}

The primary immune response involves antibodies recognizing and hinding to a foreign substance (antigen), thus targeting it for destruction. Since antigenic substances present an almost infinite variety of surface structures, the existing population of antihodies must he sufficiently varied so that there will always be some fraction with an intrinsic affinity for any particular antigen. In addition, antibodies must function in a variety of environment. e.g. in serum, tissue, and mucus membranes. Different structural classes of antihodien hate 
evolved to accomodate these requirements. The most common antibody class is Immunoglobulin $\mathrm{G}(\operatorname{IgG})$, which is a 15()-kD symmetrical $\mathrm{Y}$ - or $\mathrm{T}$ - shaped molecule with (wo) identical antigen-hinding sites approximately $100 \AA \AA$ apart at the ends of the two arms. There are a number of subclasses of $\mathrm{IgG}$, with primary structural differences occurring in the hinge region between the arms and the stem. Flexibility of the arms is postulated to he linked with differences in the ability to elicit an immune response. Knowing the details of these structures and how they relate to targeting ability of the different subclasses is fundamental to understanding the function of the antibodies. Information about the complete structure of native IgG is lacking. Small-angle X-ray and neutron solution scattering have heen performed on subclasses of IgG (Cser, Franek, Glakkikh, Kunchencko, and Ostanevich 1981), but unique structures could not be determined due to inherent problems of spherical averaging. Even fundamental quantities such as distances between antigen binding sites and the angle between the arms of the molecule are not known unequivocally.

As a class, biological experiments in solutions approaching physiological conditions are difficult because concentrations are low, the molecules are large, incoherent scattering from hydrogen is significant, and the contrast between different parts of the molecule (or macromolecular assembly) is low. On the other hand, the powerful tool of contrast matching using specific deuteration and appropriate solvent $\mathrm{D}_{2} \mathrm{O} / \mathrm{H}_{2} \mathrm{O}$ ratios can be used to enhance the signal from a particular component of the system. In this example, deuterated antigen molecules were complexed with three subclasses of proteated monoclonal IgG (identical at the atomic level) in order to determine the structural differences between the subclasses. (Sosnick, Benjamin, Novotny, Seeger, and Trewhella 1991).

Data are shown in fig. 8 for IgG1 complexed with fully deuterated nuclease, a small (17-kD) globular protein; deuteration was achieved using a bacterial expression system. The solution was $+1 \% \mathrm{D}_{2} \mathrm{O}$ to match the average scattering-length density of the antibody. The scattering pattern thus represents the shape, the mean separation, and the distribution of separations of the two antigens bound to each antibody. Data were analyzed by adjusting 


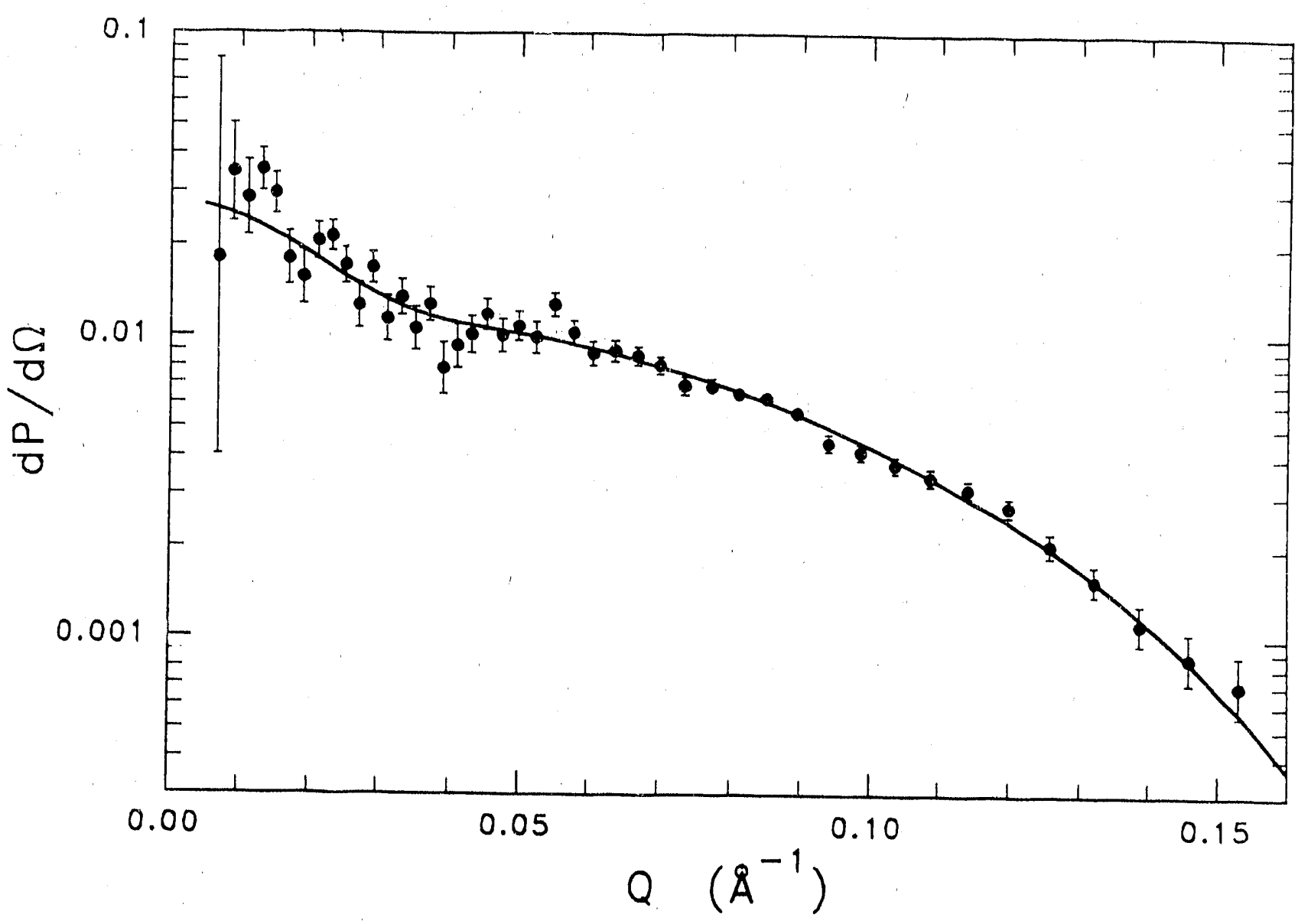

Fig. 8. Deuterated antigen complexed with contrast-matched antibody. The data have heen reduced using broader than usual $\mathrm{Q}$ bins to improve statistics. The line is a fourparameter model fit, which gives the radius of each of the two hound antigen molecules on each antibody, the distance between them, "flexibility" as represented by the polydispersity of the distance, and an overall scale factor. The average distance and flexibility were studied for several classes of antibodies. 
four model parameters to minimize the sum of residuals in data space; the model assumes the antigen molecules are spherical with radius $R$, with a mean separation $D$, and with a Gaussian distribution of separation distances representing flexibility. The fourth parameter is an overall scale factor. The line in fig. 8 is the resulting fit: $R=22.89 \pm(0.15 \AA, D=104 \pm 6 \AA$, and $\sigma=.33 \pm 4 \AA$. The reduced $x^{2}$ is 1.18 (for 40 degrees of freedom). The average distance and the flexibility are different for the three subclasses studied; these differences may now he compared to the differences in targeting and stimulating the immune system.

\section{B. Critical phenomena in polymers}

Presently there is considerable interest in the behavior of binary polymer mixtures as temperature is changed toward the critical temperature for phase separation, $T_{S}$, at the critical composition. At temperatures far from $\mathrm{T}_{\mathrm{S}}$ mean-field behavior is expected, whereas close to $T_{S}$, Ising-like behavior should occur. SANS on samples with one component deuterated can be used to study the crossover in the critical behavior. In this study (Bates, Rosedale, Stepanek, Lodge, Wiltzius, Frederickson, and Hjelm 1990) a binary mixture of lowmolecular-weight flexible polymers was used, consisting of proteated polyisoprene (PI) and partially deuterated poly(ethylene-propylene) (PEP), with $\mathrm{N}_{\text {PI }}=29$ and $\mathrm{N}_{\text {PEP }}=73$ momomers per unit. Cloud-point measurements determined the coexistence curve, establishing the critical composition at $\phi_{\mathrm{PEP}}=0.61$ and $\mathrm{T}_{\mathrm{S}} \approx 38 \mathrm{C}$. A dimensionless reduced temperature $\epsilon$ is related to the experimental temperature $T$ through the FloryHuggins interaction parameter of the components, $x: \epsilon=\left(x(T)-x_{S}\right) / x_{S}$, where $x_{S}$ is the value at $T_{S}$. The results allow an evaluation of the Ginzberg criterion that the reduced temperature $\epsilon_{x}$ at which the crossover between the two types of behavior occurs should be approximately $1 / \sqrt{ }\left(\mathrm{N}_{1} \mathrm{~N}_{2}\right)$. Although mean-field and Ising-like behavior near the critical point have been documented for binary polymer mixtures (Schwahn, Mortensen, and YeeMadeira 1987), the Ginzburg criterion has not. 
Static critical concentration susceptibility, $\kappa$, and correlation lengths, $\xi$, were studied by small-angle neutron scattering using LQD. In the single phase region near the critical point the scattering intensity $\mathrm{I}(\mathrm{Q})$ can be expanded at small momentum transfer in an Ornstein-Zernike relationship, $\mathrm{I}(\mathrm{Q})=\mathrm{I}(0) /\left(1-\mathrm{Q}^{2} \xi^{2}\right)$, and $\kappa \propto \mathrm{I}(0)$. Both $\kappa$ and $\xi$ diverge at small values of $\epsilon$, respectively as $\epsilon^{-\gamma}$ and $\epsilon^{-\nu}$. For mean-field behavior $\gamma=1.0$ and $\nu=0.50$, whereas for Ising-like behavior the expectations are $\gamma=1.26$ and $\nu=0.63$. Thus the change in the dependence of $I(0)$ and $\xi$ on $\epsilon$ is used to determine the crossover.

Representative Ornstein-Zernicke plots and the resultant $1 / \mathrm{I}(0)$ vs. $1 / \mathrm{T}$ plot are shown in fig. 9. These static measurements for $\mathrm{I}(0)$ (and similarly for $\xi$ ) yielded a crossover point at about $70 \mathrm{C}$ or $\epsilon_{\mathrm{x}}=0.11$, which is different by a factor of 40 from that predicted by the criterion above, which assumed $\mathrm{N}_{1} \approx \mathrm{N}_{2}$. For asymmetric mixtures, the appropriate Ginzburg criterion is

$$
\epsilon_{\mathrm{x}}=\mathrm{Cv}_{\mathrm{m}}^{2} \frac{\left[\mathrm{N}_{1}^{-1} \varphi_{1}^{-3}+\mathrm{N}_{2}^{-1}\left(1-\varphi_{1}\right)^{-3}\right]^{2}}{\left[\mathrm{~N}_{1}^{-1} \varphi_{1}^{-1}+\mathrm{N}_{2}^{-1}\left(1-\varphi_{1}\right)^{-1}\right]\left[\mathrm{R}_{1}^{2} \mathrm{~N}_{1}^{-1} \varphi_{1}^{-1}+\mathrm{R}_{2}^{2} \mathrm{~N}_{2}^{-1}\left(1-\varphi_{1}\right)^{-1}\right]^{3}}
$$

Here $R_{i}$ is the radius of gyration of polymer $i$ (assuming a Gaussian coil), and $v_{m}$ is the monomer volume. Eq. (5) includes effects both for $N_{1} \neq N_{2}$ and $R_{1} \neq R_{2}$. With $C=$ $0.29 \pm 0.08$ (derived from measured radii of gyration, $N$ and $\epsilon_{x}$ ), this expression for $\epsilon_{x}$ agrees with the experimental value to within the experimental uncertainty. This is the first verification of the Ginzburg criterion for low-molecular-weight, flexible polymers.

\section{Ternary surfactant liquid crystals}

Cubic liquid crystalline phases formed by amphiphiles in the presence of water have been known for some time and extensively studied. More recently, similar phases in ternary surfactant systems have been studied. These consist of bicontinuous periodic labyrinths of water and oil having cubic symmetry. In recent work conducted on LQD and D-17 (at ILL) and also using small-angle X-ray scattering (Radiman, Toprakcioglu, Dai, Faruqi, Hjelm, and 


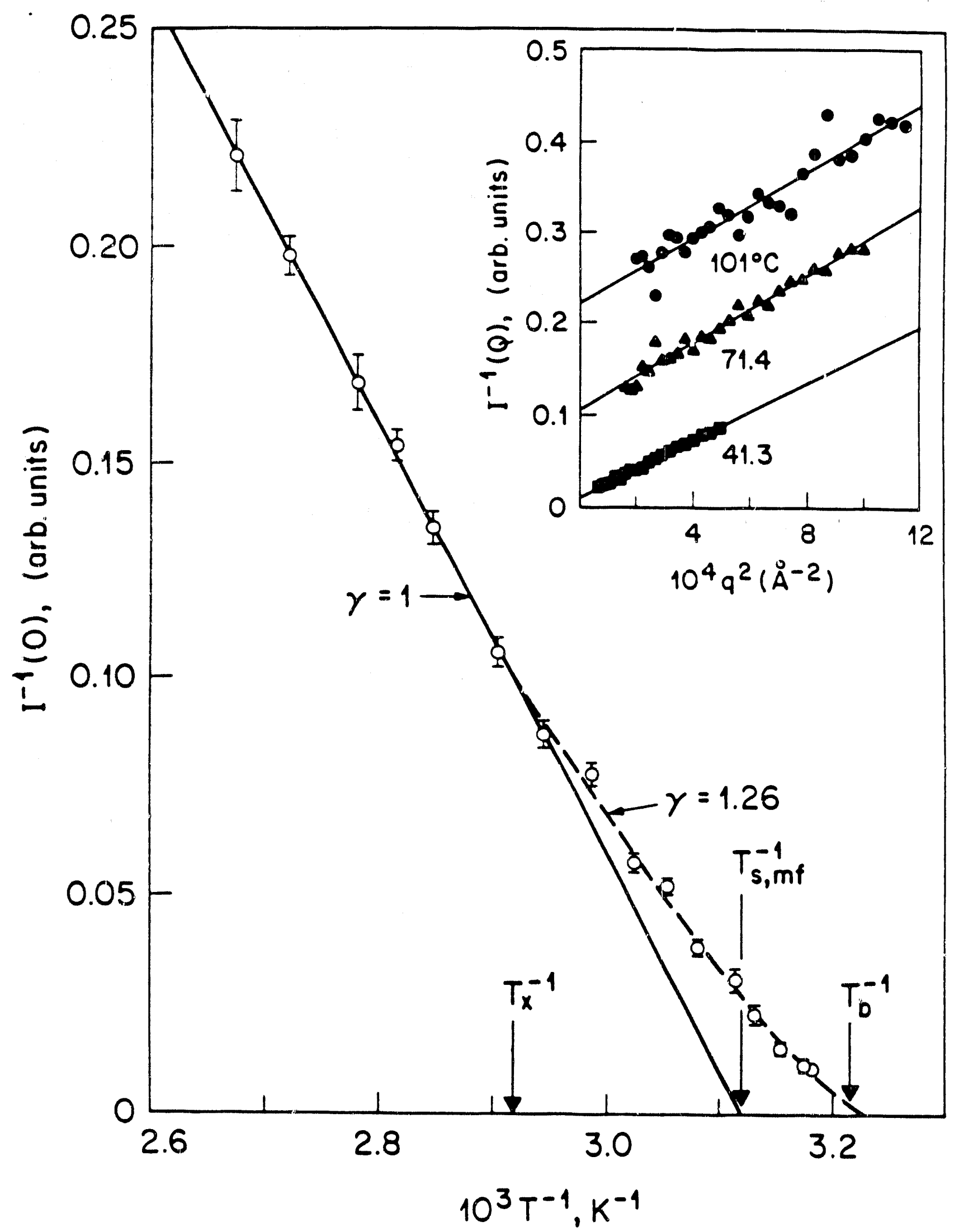

Fig. 9. Inverse susceptibility 's. inverse temperature for low-molecular-weight polymer hlend of polyisoprene and poly(ethylene-propylene). Ornstein-Zernicke plots of typical data are shown in the inset. Crossover from mean-field to Ising-like behavior is evidenced by deviation from linearity. Figure taken from Bates et al. (1990). 
de Vallera 1990), the cubic phases of a ternary mixture of $D_{2} \mathrm{O}$, didodecyldimethylammonium bromide (DDAB) and octane where studied. Samples containing a constant ratio of DDAB and octane where diluted from $\phi\left(\mathrm{D}_{2} \mathrm{O}\right)$ about 0.38 to 0.65 , thus sampling most of the cubic phase region of the system. A symmetry transition from a diamond-D structure to bcc was observed in SAXS data when the aqueous volume fraction reached 0.55 .

Because only a small fraction of the neutrons within one time slice satisfy the Bragg condition for a specific lattice point in reciprocal space, powder-pattern intensities must 'ee corrected by a Lorentz factor which is the ratio of the "dwell time" of hie collapsing Ewald sphere to the width of the time slice. In general, the velocity of the surface is a function of wavelength and angle. The radius of the sphere is $1 / \lambda$, which by eq. (1) is proportional to $1 / i$. Thus the rate of collapse is proportional to $1 / \lambda^{2}$. Since the origin of reciprocal space is also on the Ewald sphere, the whole sphere is translating toward the origin with the same speed as the collapse. Thus every point on the Ewald sphere is moving toward the origin with a velocity proportional to $(\sin \theta) / \lambda^{2}$, and the relative dwell time is $\lambda / Q$. Since the time slices are logarithmic, i.e. also proportional to $\lambda$, the correction term is only a factor of $Q$; this is very fortunate because if there were separate angle- or wavelength-dependent factors they would have to be applied before rebinning into $\mathrm{Q}$.

Figure 10 shows SANS data from LQD for a typical sample with bcc symmetry, multiplied by $Q$ to give a relative intensity scale. Calculations of the reduced area of interface per unit cell support the notion that the surfactant forms inio a bilayer in both symmetries. However, the SANS data for the bcc structure are not consistent with this conclusiun, as the strengths of the observed diffraction peaks (as seen in fig. 10) are inconsistent with a theoretical prediction that the (200) reflection should be weaker than the (211) for a bilayer: thus a monolayer structure is suggested for the bcc structure. This example shows the importance of comparison of the results of SAXS and SANS in such systemis. 


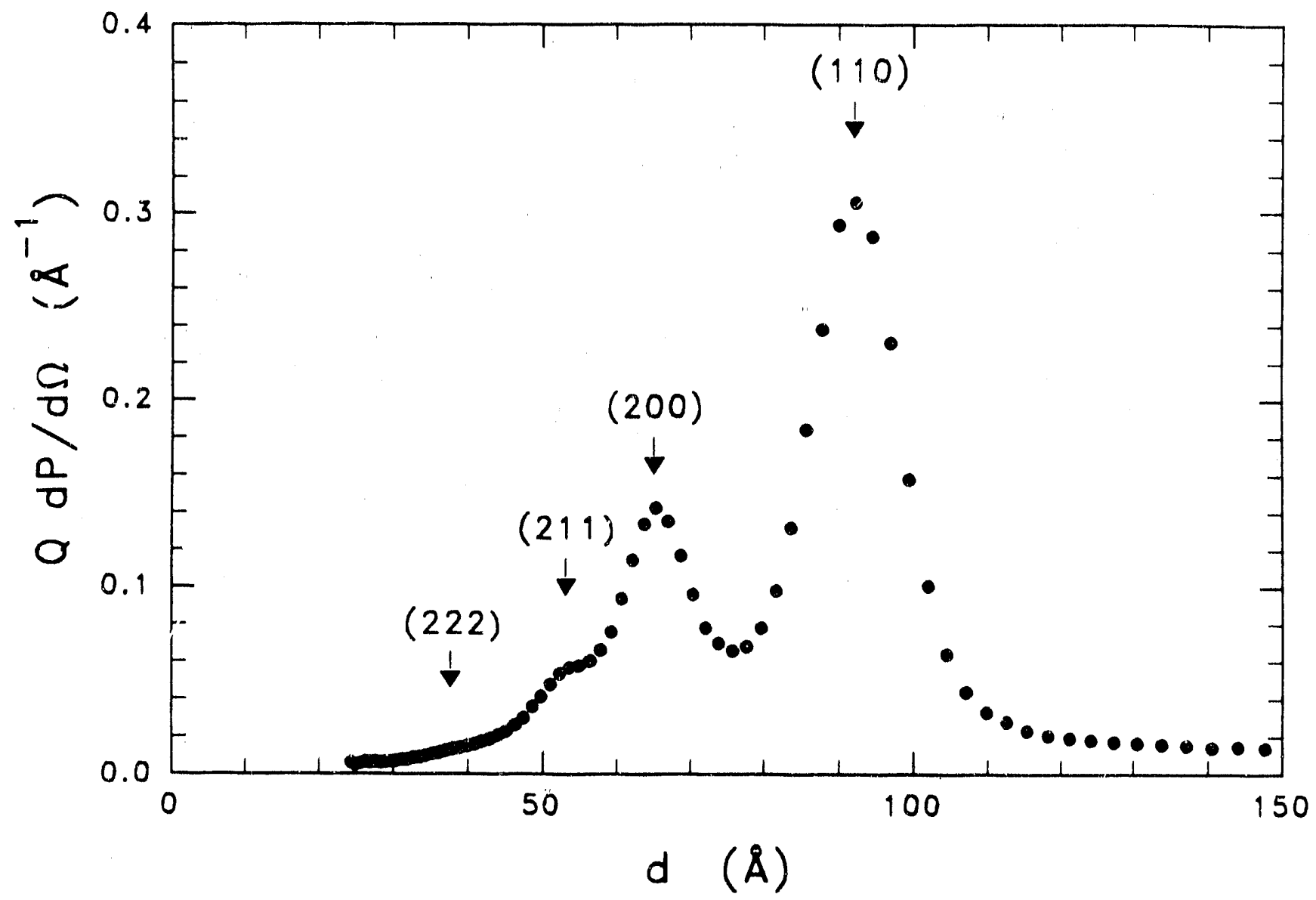

Fig. 10. Low-resolution powder diffraction pattern of ternary surfactant liquid crystal. The sample is $36.22 \%$ DDAB (didodecyldimethylammonium bromide), $56.36 \% \mathrm{D}_{2} \mathrm{O}$, and $7.42 \%$ octane. The observed Bragg reflections correspond to bcc symmetry (i.e., the sums of the indices are even) with a unit cell dimension of $128 \AA$. The Q-range represented is $0.25 \AA^{-1}$ to $0.04 \AA^{-1}$. 


\section{Compressibility of fumed silica powders}

Fumed silica ("glass soot") consists of submicron fractal aggregates of random, weakly branched strings of 100$)-\AA$ silica spheres, with a great deal of internal porosity. Mercury porosimetry and mechanical compressibility measurements yield the empirical relationship that the pore volume $V_{p} \propto P^{-1 / 3}$, where the pore volume is the total sample volume less the incompressible silica volume $\mathrm{V}_{\mathrm{s}}$, and $\mathrm{P}$ is the applied pressure. Thus the compressibility $\kappa$ is found to be inversely proportional to P. A preliminary reactor neutron scattering study on compacted fumed silica powders demonstrated that the forward scattering $I(0)$ decreases with increasing compression. This suggested the possibility that $I(C) \propto \kappa$, which would imply a straightforward statistical interpretation of the pressure-volume relationship, mimicking systems in thermodynamic equilibrium. If the particles are sufficiently numerous and the local construction rules well defined, then the macroscopic properties of the powder should be predictable and interesting (Edwards and Oakeshott 1989). Essentially, all configurations consistent with mechanical stability are equally probable, but the measurable properties are essentially the same for the overwhelming majority of configurations. For example, the volume of a heap of sand shou'd be predictable. Unfortunately, to extend the thermodynamic analogy further and develop a pressure-volume equation of state, one must understand the rôle of energy in the powder system, and derive the proper analogue of the Helmholtz free energy. The fate of the mechanical energy put into the system during compression has not yet been identified. A more precise experimental determination of the relationship between forward scattering intensity and the compressibility should help clarify the process.

Samples of Cab-O-Sil fumed silica (grade EH-5, Cabot Corporation) were prepared in closed aluminum cells with 1-mm thick aluminum windows and 1-mm internal path length. Densities in the current study ranged from $0.1 \mathrm{~g} / \mathrm{cm}^{3}$ to $1.1 \mathrm{~g} / \mathrm{cm}^{3}$, supplementing the earlier data at densities down to $0.04 \mathrm{~g} / \mathrm{cm}^{3}$ obtained during the 1989 LANSCE running cycle (Hurd, Johnson, and Smith 199()). Scattering from an empty cell was subtracted from each data set, and a small incoherent contribution proportional to the mass of the sample was also 
subtracted. Data over the extended high-Q range were fitted by a power law to confirm the fractal nature of the aggregates; the fractal dimension (6-power-law exponent) varied very little with density, and had an average value of 2.5. There is some indication of additional short-range structure in the scattering curves for densities greater than $\left(0.5 \mathrm{~g} / \mathrm{cm}^{3}\right.$. To obtain an estimate of zero-angle scattering probability, $\mathrm{dP}(0) / \mathrm{d} \Omega$, the low-Q data were fitted with a two-parameter Debye-Bueche random-coil model by plotting $[\mathrm{dP}(\mathrm{Q}) / \mathrm{d} \Omega]^{-1 / 2}$ vs. $\mathrm{Q}^{2}$. The slope of this plot is the square of the correlation length $\xi$, which should decrease with compression. For densities $\geq 0.1 \mathrm{~g} / \mathrm{cm}^{3}$, good linear fits were obtained in the regime $\mathrm{Q} \xi<1$, with $\xi$ varying from $140 \AA$ down to $60 \AA$ for the most compressed samples. The fits to lowerdensity samples are less satisfactory because the Q-range of LQD does not extend low enough to guarantee that we actually have data for $Q \xi<1$. Therefore only the data from the current cycle (Hurd, Johnston, Smith, and Seeger 1991) are included in the analysis.

The resulting $d P(0) / d \Omega$, normalized by dividing by sample thickness and density, are shown in fig. 11 as a function of $\mathrm{V}_{\mathrm{p}} / \mathrm{V}_{\mathrm{s}}$, where $\mathrm{V}_{\mathrm{s}}$ was computed assuming a density of $2.2 \mathrm{~g} / \mathrm{cm}^{3}$ for the silica particles. If $\mathrm{I}(0) \propto \propto \propto \mathrm{V}_{\mathrm{p}}^{3}$, then the slope in fig. 11 would be approximately 3 . The observed power law is however only 1.14 , clearly disproving the hypothesis. This failure does not mean that there is no ergodic analogue for fumed silica powders, but merely that the large-scale fluctuations which lead to small-angle scattering do not control the compressibility in the same way as in thermal systems.

\section{Acknowledgements}

We wish to thank the various experimenters and authors for use of published and unpublished data, and for their universal patience and understanding as operating and datareduction procedures evolve. This work was performed under the auspices of the Office of Basic Energy Sciences, U. S. Department of Energy, under contract no. W-7405-ENG-32 to the University of California. 


\section{References}

Bates, F. S., Rosedale, J. H., Stepanek, P., Lodge, T. P., Wiltzius, P., Fredrickson, G. H., \& HJElM, R. P. JR (199()). Phys. Rev. Lett. 65, 1893-1896.

Borso, C. S., Carpenter, J. M., Williamson, F. S., Holmblad, G. L., Mueller, H., Faber, J. JR, Epperson, J. E., \& Danyluk, S. S. (1982). J. Appl. Cryst. 15, 443-448.

Convert, P. \& Forsyth, J. B. (1983). Position-Sensitive Detection of Thermal Neutrons. London: Academic Press.

Crawford, R. K. \& Carpenter, J. M. (1988). J. Appl. Cryst. 21, 589-6()1.

CRAWfORd, R. K., EpPerson, J. E., \& ThIYAgarajan, P. (1989). Advanced Neutron Sources 1988, Institute of Physics Conference series 97, edited by D. K. HYER, pp. 419-426. Bristol, IOP Publishing.

EDWARDS, S. F. \& OAKESHOTT, R. B. S. (1989). Physica D 38, 88- .

Cser, L., Franek, F., Glakkikh, I. A., Kunchencko, A. B., \& Ostanevich, Y. M. (1981). Eur. J. Biochem. 116, 109-116.

Epperson, J. E., Worcester, D. L., Thiyagarajan, P., Klippert, T. E., Crawford, R. K., \& CARPENTER, J. M. (1987). Unpublished. See Instrumental Techniques and Dota Analysis at Pulsed Source SANS Instruments, edited by R. K. CRAWFORD, J. E. EPPERSON, P. ThIYAgarajan, \& F. TROUw, IPNS Note 46, pp. 33-34. Argonn National Laboratory, Argonne, IL, USA.

Glinka, C. J., Rowe, J. M., \& LaRock, J. G. (1986́). J. Appl. Cryst. 19, 427-439.

HEENAN, R. (1987). Instrumental Techniques rind Data Analysis at Pulsed Source SANS Instruments, edited by R. K. CRAWFORD, J. E. EPPERSON, P. THIYAGARAJAN, \& F. TROUW, IPNS Note 46, pp. 11-14. Argonne National Laboratory, Argonne, IL, USA.

HJELM, R. P. JR (1987). J. Appl. Cryst. 20, 273-279. 
HJELM, R. P. JR (1988). J. Appl. Cryst. 21, 618-628.

HJelm, R. P. JR \& SEeger, P. A. (1989). Advanced Neutron Sources 1988, Institute of Physics Conference series 97, edited by D. K. HYER, pp. 367-387. Bristol, IOP Publishing.

Hurd, A. J., Johnston, G. P., \& SMith, D. M. (1990). Proceedings of COPS II, Alicante, Spain, May 6-9, 1990, edited by F. RodRIGUES-REINOSO. In press.

Hurd, A. J., Johnston, G. P., SMIth, D. M., \& SEeger, P. A. (1991). LANSCE Progress Report (unpublished).

Ishikawa, Y., Furusaka, M., Nimmura, N. Arai, M., \& Hasegawa, K. (1986). J. Appl. Cryst. 19, 229-242.

KiYanagi, Y. \& Watanabe, N. (1990)). Proceedings of the 11th Meeting of the International Collaboration on Advanced Neutron Sources, October 22-26, 1991), Tskuba, Japan.

McElhaney, S. A. \& Vandermolen, R.I. (1990). Two-Dimensional Position-Sensitive Detectors for Small-Angle Neutron Scattering, ORNL/TM-11557, Oak Ridge National Laboratory, Oak Ridge, TN, USA.

Mildner, D. F. R. \& CARPenter, J. M. (1984). J. Appl. Cryst. 17, 249-256.

Radiman, S., Toprakcioglu, C., Dai, L., Faruqi, A. R., HJelm, R. P. JR, \& DE VAllera, A., (1990). J. de Phys. France, in press.

Russell, G. J. (1990). Proceedings of the 11th Meeting of the International Collaboration on Advanced Neutron Sources, October 22-26, 1990, Tskuba, Japan.

Russell, G. J., Gilmore, J. S., Robinson, H., Legate, G. L., Bridge, A., Sanchez, R. J., BREWTON, R. J., WOODS, R., \& HUGHES, H. G. III (1989). Advanced Neutron Sources 1988, Institute of Physics Conference series 97, edited by D. K. HYER, pp. 483-496. Bristol, IOP Publishing. 
Schwahn, D., Mortensen, K., \& Yee-Madelira, Y. (1987). Phys. Rev. Lett. 58, 15441546.

SEEGER, P. A. (1980). Nucl. Instrum. Methods 178, 157-161.

SEEgER, P. A. (1984). IEEE Trans. Nucl. Sci. NS-31, 274-280.

SeEger, P. A. (1986). Physica (Utrecht) 136B, 106-109.

SEeger, P. A. (1988). J. Appl. Cryst. 21, 613-617.

Seeger, P. A., HJelm, R. P. JR, \& Nutter, M. J. (1990)). Mol. Cryst. Liq. Cryst. 180A, 101-117.

SEEger, P. A. \& PYNN, R. (1986). Nucl. Instrum. Methods A245, 115-124.

Seeger, P. A., Williams, A., \& Trewhella, J. (1986). Proceedings of Internationai Collaboration on Advanced Neutron Sources-VIII, 8-11 July, 1985, Oxford. Report RAL-85-110, vol. II, pp. 441-453. Rutherford Appleton Laboratory, Didcot, England.

Sosnick, T., Benjamin, D., Novotny, J., Seeger, P. A., \& Trewhella, J. (1991). Biophysical Society, San Francisco, Feb. 24-28. 

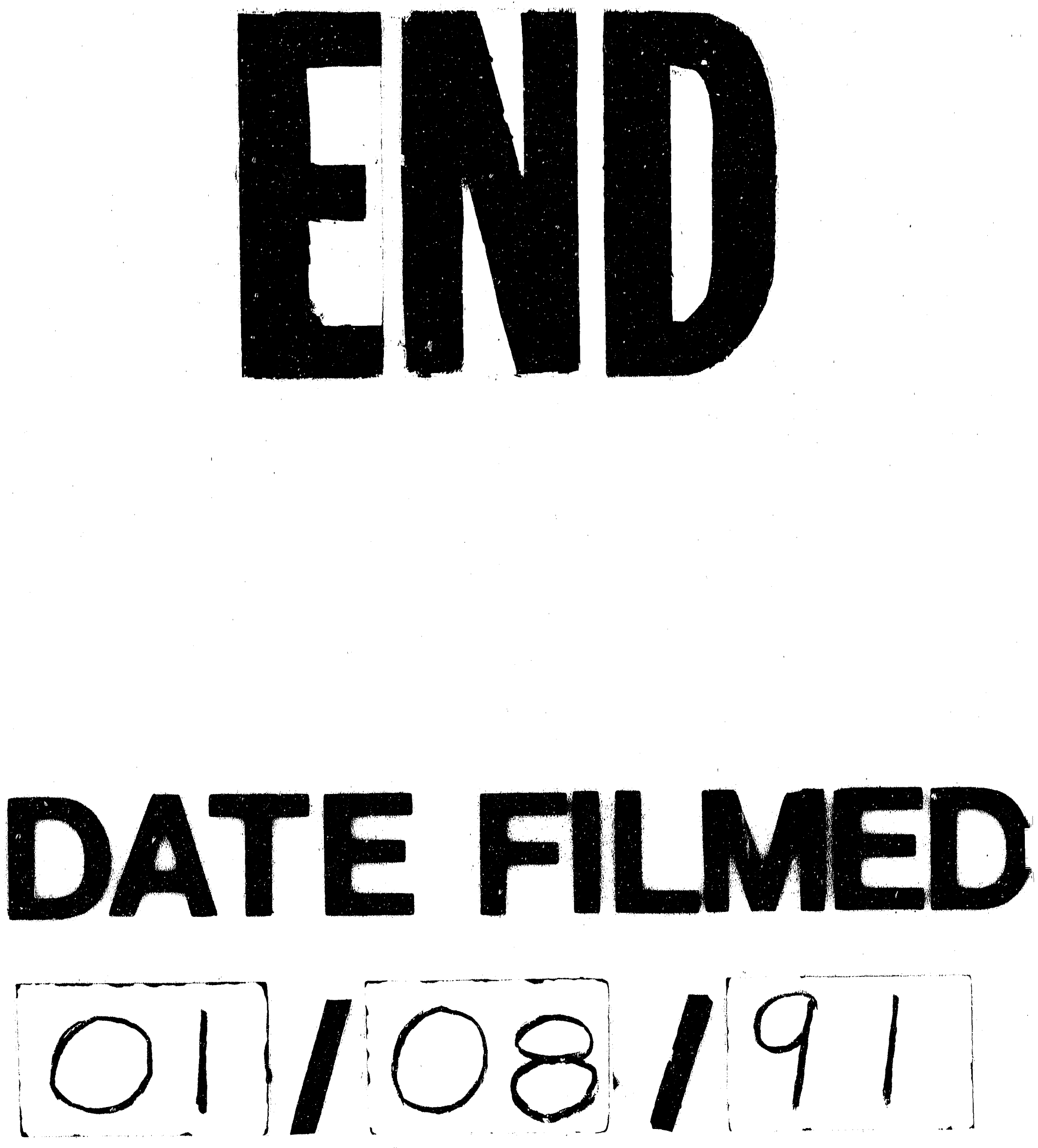
